Accepted Manuscript

This is an Accepted Manuscript of the following article:

J.A. Baz-Lomba, Malcolm J. Reid, Kevin V. Thomas. Target and suspect screening of psychoactive substances in sewage-based samples by UHPLC-QTOF. Analytica Chimica Acta.

Volume 914, 2016, pages 81-90, ISSN 0003-2670.

The article has been published in final form by Elsevier at http://dx.doi.org/10.1016/j.aca.2016.01.056

(C) 2016. This manuscript version is made available under the CC-BY-NC-ND 4.0 license http://creativecommons.org/licenses/by-nc-nd/4.0/

It is recommended to use the published version for citation. 


\section{Target and suspect screening of psychoactive substances in sewage-based samples 2 by UHPLC-QTOF}

3 J.A. Baz-Lomba ${ }^{\text {a,b }}$, Malcolm J. Reid ${ }^{\text {a }}$, Kevin V. Thomas ${ }^{\text {a }}$

$4 \quad{ }^{a}$ Norwegian Institute for Water Research, Gaustadalléen 21, NO-0349 Oslo, Norway

5 bFaculty of Medicine, University of Oslo, PO box 1078 Blindern, 0316 Oslo, Norway

\section{Abstract}

7 The quantification of illicit drug and pharmaceutical residues in sewage has been shown to be a valuable tool that complements existing approaches in monitoring the patterns and trends of drug use. The present work delineates the development of a novel analytical tool and dynamic workflow for the analysis of a wide range of substances in sewage-based samples. The validated method can simultaneously quantify 51 target psychoactive substances and pharmaceuticals in sewage-based samples using an off-line automated solid phase extraction (SPE-DEX) method, using Oasis HLB disks, followed by ultra-high performance liquid chromatography coupled to quadrupole time-of-flight mass spectrometry (UHPLC-QTOF) in MS ${ }^{\mathrm{e}}$. Quantification and matrix effect corrections were overcome with the use of 25 isotopic labeled internal standards (ILIS). Recoveries were generally greater than $60 \%$ and the limits of quantification were in the low nanogram-per-liter range (0.4-187 ng L ${ }^{-1}$ ). The emergence of new psychoactive substances (NPS) on the drug scene poses a specific analytical challenge since their market is highly dynamic with new compounds continuously entering the market. Suspect screening using high-resolution mass spectrometry (HRMS) simultaneously allowed the unequivocal identification of NPS based on a mass accuracy criteria of $5 \mathrm{ppm}$ (of the molecular ion and at least two fragments) and retention time (2.5\% tolerance) using the UNIFI screening platform. Applying $\mathrm{MS}^{\mathrm{e}}$ data against a suspect screening database of over 1,000 drugs and metabolites, this method becomes a broad and reliable tool to detect and confirm NPS occurrence. This was demonstrated through the HRMS analysis of three different sewage-based sample types; influent wastewater, passive sampler extracts and pooled urine samples resulting in the concurrent quantification of known psychoactive substances and the identification of NPS and pharmaceuticals.

\section{Keywords}

27 High resolution mass spectrometry, Sewage-base epidemiology, Multi-residue method, Dynamic workflow, Screening, Psychoactive drugs 


\section{Introduction}

30 The illicit use of psychoactive substances is a worldwide phenomenon with incalculable consequences on society. Estimates from 2012, indicate that between the 4 and $7 \%$ of the world's population has used an illicit substance at least once during the previous year [1]. Even though the overall European drug situation is generally stable, a graduated and complex situation is emerging [2]. Classic drugs, such as heroin, cocaine and cannabis remain dominant but the drug market is now peppered with a vast array of synthetic psychoactive substances. The EU Early Warning System for first time notified the discovery of 101 new psychoactive substances (NPS) in 2014 [3]. Illicit drug use and prevalence estimations are typically based on population surveys, clinical cases, seizures and mortality rates related to use. In addition to known problems with bias and a lack of representativeness, these approaches also require a long time to establish a general overview of the illicit drug situation and, generally, results are published several years post survey [4].

40 Pharmaceuticals arising from human, veterinary and aquaculture use, occur in the aquatic environment as one of the largest sources of contaminants of anthropogenic origin and have recently become a new environmental problem [5]. Many studies have been performed assessing the impact of hospitals and their contribution to the pharmaceutical loads found in wastewater [6-8], the efficiency of the removal of pharmaceuticals by wastewater treatment plants (WWTP) [9], the spatial and temporal differences of pharmaceuticals occurrence in wastewater [10] and recently to compare the results of the wastewater analysis with predicted concentrations based on prescription figures $[11,12]$. In the context of sewage-based epidemiology (SBE) pharmaceutical residues also offer the potential to map certain types of disease and other health based stressors[13].

The measurement of illicit drugs and pharmaceuticals in wastewater has become an extended topic over the past decade and SBE has been established as a complementary approach for monitoring trends of illegal drug use [14-18]. Recently the approach has been evaluated with an international study comparing the drug consumption in 21 different countries by quantitatively analyzing illicit drugs and their metabolites in 47 WWTP $[19,20]$. SBE has been used to estimate drug use in communities (i.e. city, small town, school, prisons) as well as identify changes in drug consumption during special events [21, 22], temporal changes [17], and providing complementary data on the use of NPS [23-25]. Thus far, the analytical methods used for SBE have focused on the principal challenges related to the low concentrations of drug residues in wastewater combined with the complexity of the wastewater matrix. These procedures have been mainly developed for classic illicit drugs (cocaine, amphetamines, opioids and cannabis) and a broad range of pharmaceuticals, and based on solid-phase extraction (SPE) for sample pre-treatment and pre-concentration followed by instrumental analysis, typically liquid chromatography coupled to tandem mass spectrometry (QqQ) $[5,16]$. 
The hyphenation of liquid chromatography to high resolution mass spectrometry (HRMS), i.e. orbitrap and quadrupole time-of-flight (QTOF), has increased the potential of SBE to identify NPS and their residues in sewage based samples. HRMS offers some unique advantages for screening and identification, such as better specificity due to its increased resolving power and the possibility to perform retrospective analyses without the need of additional sample analyses [26].

Since the NPS market is in constant flux, new strategies are urgently required. New analytical methods based on QqQ for the target analysis of NPS are continuously being published[24, 25] but in general these techniques cannot keep up with the rapidly evolving NPS market making necessary the development of new approaches that combine target analysis with wide-scope suspect screening capable of confirming the presence/absence of a new compounds with quantification should reference standards be available[27]. This challenge can be overcome by using a suspect screening approach that has already been demonstrated as a suitable technique for the detection and confirmation of several organic compounds[27], including NPS[23, 28]. The simultaneous determination of a broad number of compounds in one injection, with a corresponding reduction of time and costs, without the need for reference standards make this approach one of the current trends in environmental analytical chemistry.

The aim of the presented work was to establish a broad analytical tool for the analysis of different types of drugs in sewage-based samples, such as pooled urine, wastewater and passive sampler extracts and develop a dynamic workflow for the introduction of new substances of interest in the future due to the high potential of the HR-MS instruments for the identification of suspect compounds presented in the sample.. Firstly a targeted multi-residue method for the simultaneous identification and quantification of 51 drugs, pharmaceuticals and their main urinary metabolites by UHPLC/QTOF was validated as recommended by EU guidelines with minor modifications $[29,30]$. With the target method established to track the quantitative occurrence of selected target analytes, the secondary goal of this work was to detect possible NPS and other compound of possible interest in sewage-based samples thought the use of a suspect screening approach. The present work also describes the potential of non-target analysis for identifying NPS and their subsequent inclusion into the suspect database or target method if standard reference is available through a dynamic approach.

\section{Materials and methods}

87 Detailed information relating to reagents and chemicals (illicit drugs, pharmaceuticals and metabolite reference substances), the sewage-based samples and extraction procedure can be found in the Supplementary Information (SI). 
Influent samples were collected from two Norwegian WWTPs; VEAS WWTP in Oslo and Ladehammeren and

Høvringen WWTP in Trondheim. The robustness of the method was also examined by analysing different sewage-based samples. The "Pharmaceuticals" version of the polar organic chemical integrative sampler (POCIS) was placed for two weeks in the sedimentation overflow channel at the Oslo WWTP and the pooled urine samples were collected from three different Norwegian music festivals. Figure 1 shows an overview of the samples used in this study (detailed information provided in SI).

\subsection{Extraction procedure}

Defrosted sewage samples $(100 \mathrm{~mL})$ were spiked with a mix of 25 isotope labeled internal standards (ILIS) to

100 give a concentration of $400 \mathrm{ng} \mathrm{L}^{-1}$. The $\mathrm{pH}$ was controlled but generally was the desired $(\sim 7)$. Subsequently, extraction was performed using a SPE-DEX fully automatable extraction system (Horizon Technology, Salem,

102 NH, USA) using HLB (Hydrophilic-Lipophilic Balance) extraction disks (47 mm, I.D.; Horizon Technology,

103 City, Country) (more information in SI and Table S-1).

104 The selection of the Oasis HLB extraction sorbent was based on previous experience. Oasis HLB and MCX

105 generally yield the highest recoveries for most of the drugs and pharmaceuticals [5, 18]. Compared with Oasis

106 MCX, HLB provides a lower selectivity for some basic compounds, such as amphetamine-like compounds and

107 a considerable increase of the matrix components retained in the sorbent leading to a higher sensitivity. Despite

108 this, HLB, with a mixed-mode cation exchange sorbent, offers the possibility to extract a wide range of 109 compounds with different psychochemical characteristics suiting the goal of the method, enabling the

110 simultaneous analysis of the widest range of drugs and pharmaceuticals in one single extraction.

111 To determine the method recovery influent wastewater from VEAS WWTP in Oslo was spiked in quintuplicate

112 with a standard mixture containing all of the target analytes at two different concentrations together with a

113 mixture of the 25 ILIS. The two concentrations were selected according to those generally found in wastewater 114 influent.

\subsection{Ultra-high pressure liquid chromatography}

116 The chromatography column, stationary phase and mobile phases were selected in accordance with a pre117 established screening method[31]. Figure 2 shows the 2D mass chromatogram of the low energy channel (6eV $118 \mathrm{ESI}^{+}$) for the optimal separation of all target compounds and NPS at a concentration of $250 \mathrm{ng} \mathrm{mL}^{-1}$. A Waters 119 Acquity UPLC system (Milford, MA, USA) was used for this work. Chromatographic separation was carried 120 out using an Acquity UPLC HSS C18 column (2.1 x $150 \mathrm{~mm}$, particle size $1.8 \mu \mathrm{m})$ (Waters, Milford, MA, 121 USA). Gradient elution was performed at a constant flow of $0.4 \mathrm{ml} \mathrm{min}^{-1}$ using $5 \mathrm{mM}$ ammonium formate, $\mathrm{pH}$ 
1223.0 (solvent A) and acetonitrile with $0.1 \%$ formic acid (solvent B). The gradient elution starts with $87 \%$ A and

123 then increasing B to $95 \%$ in 15 minutes: Solvent A, held for $0.5 \mathrm{~min}$; 0.5-10 linear rate to $50 \% \mathrm{~B}, 10-10.75$

124 linear rate to $95 \% \mathrm{~B}$, held for $0.5 \mathrm{~min}$; reconditioning with a linear rate to $87 \% \mathrm{~A}, 12.50-15 \mathrm{~min}$. The analytical

125 column and the guard column were kept at $50^{\circ} \mathrm{C}$ and the sampler manager at $5{ }^{\circ} \mathrm{C}$. The weak and strong wash

126 used to remove the contaminants from the needle and the injection port were $10 \% \mathrm{ACN}$ in water $(600 \mu \mathrm{L})$ and

$12795 \% \mathrm{ACN}$ in water $(200 \mu \mathrm{L})$ respectively.

128 2.4 Quadrupole Time-of-flight Mass Spectrometry

129 A Xevo G2-S Q-TOF mass spectrometer (Waters, Milford, MA USA) was used in positive ESI mode for 130 acquisition using $\mathrm{MS}^{\mathrm{e}}$, that allows both precursor and product ion data to be simultaneously acquired during a

131 single run. The MS method consists of 3 functions, the first (low energy, LE) applies collision energy of $6 \mathrm{eV}$,

132 the second function (high energy, HE) acquires through a collision energy ramp of 15-50 eV and the third

133 function acquires the lock mass data for online mass calibration. The MS range is 70-700 with a scan time of

1340.1 second in continuum mode, preserving the peak shape of the exact-mass precursor and product ions. The

135 source conditions whose maximum intensities were achieved were the following: capillary voltage $3 \mathrm{kV}$,

136 sample cone, $20 \mathrm{~V}$, source offset $80 \mathrm{~V}$, source temperature $120^{\circ} \mathrm{C}$, desolvation temperature $500{ }^{\circ} \mathrm{C}$, cone gas

137 flow rate $50 \mathrm{~L} \mathrm{~h}^{-1}$, desolvation gas $\left(\mathrm{N}_{2}\right)$ flow rate $1000 \mathrm{~L} \mathrm{~h}^{-1}$.

138 The mass spectrometer was calibrated using a solution of sodium formate over a mass range of 50-1000 Da.

139 Analyses were performed using an external reference (Lock-Spray ${ }^{\mathrm{TM}}$ ). During the data acquisition the mass

140 was corrected using an external reference (Lock-Spray ${ }^{\mathrm{TM}}$ ) consisting of $0.2 \mu \mathrm{g} \mathrm{mL}^{-1}$ solution of leucine-

141 enkephalin infused continuously at $10 \mu \mathrm{L} \mathrm{min}^{-1}$ via a lockspray interface. The lock mass data were acquired

142 every 20 seconds for 0.1 seconds and for the rest of the time the baffle in the ion source blocked the entry of

143 the lock spry. This generated a reference ion in positive mode at $\mathrm{m} / \mathrm{z} 556.2771$ that was used for real-time mass

144 corrections in order to maintain the mass accuracy and reproducibility.

$145 \quad 2.5$ Data processing

146 The workflow and identification confidence used in this work were based upon those described by Krauss et al.

147 [32] and Schymanski et al. [33](Figure 3).

148 The MSe data processing using the UNIFI screening platform (Waters Corporation, Milford MA, USA) was

149 performed in two steps. Firstly, all the continuum data was peak detected using a 3D peak algorithm based on

150 the calculation of the peak volumes by the detection of all the ion crests in a given mountain range. This

151 provides a complete list of retention-time $/ \mathrm{m} / \mathrm{z}$-pairs which are then used for screening. The second step which

152 follows peak detection is the screening protocol where Unifi matches the observed $\mathrm{m} / \mathrm{z}$ retention-time pairs

Page 5 of 22 
154 of parent ion and fragment ions $(5 \mathrm{ppm})$ and theoretical isotope patterns for the protonated molecule at LE and at least two accurate fragment ions at HE [30]. The database is built from a pre-established screening method

156 [31] which uses the same chromatographic and detector parameters as in this study.

157 For target screening the procedure is unchanged, except the screening is focused only on the 51 target 158 compounds (as opposed to the entire $1000+$ library) and reference standards are run with the analysis to prove quantitative information/results (Table 1). Protonated precursor ion data at LE was extracted and used for

160 quantification. For this work, selection of the 51 target analytes was based on their reported use in Norway and

161 their previously reported occurrence in wastewater [17, 19, 24].

162 For suspect screening, the retention-time/ m/z-pairs are compared against a broad list of approximately 1000

163 compounds (note that this database is under constant renewal and expansion so the exact number of compounds 164 varies over time). UNIFI also automatically assesses the candidate values with the fragments, mass defect, 165 isotope pattern and adducts $\left(\mathrm{M}+\mathrm{H}, \mathrm{M}+\mathrm{Na}, \mathrm{M}+\mathrm{NH}_{4}\right)$. The suspect screening database (including mass spectra, 166 assigned fragments and retention time) was applied under the same acquisition parameters as above and tested 167 in this work on pooled urine samples in an attempt to identify NPS or possible compounds of interest to include 168 in the future in the target method through a reiterative development workflow.

169 While the current study used a screening database developed around a commercially product, and manually 170 augmented with 16 NPS, suspect screening may also be performed using other public databases (e.g. 171 MassBank, Chemspider) or in-silico fragmentation tools (MetFrag) which are available for many 172 environmental contaminants. Suspect screening lists may also be produced with computational prediction 173 systems that can provide a list of potential excreted biomarkers and biotransformation products in wastewater 174 [34].

175 Directly infusing the standards and subsequently analyzing the spectra usually calculate fragmentation 176 pathways. This study attempts to complement the suspect-screening database by importing suspect candidates 177 relying on accurate spectral information and different non-target tools. Where analytical data on fragmentation 178 is lacking the UNIFI fragment match tool applies a series of novel algorithms based on systematic bond 179 disconnections of the precursor structure to predict fragmentation pathways that can then be compared with 180 measured spectra in order to help to increase the amount and the reliability of the information essential for the 181 subsequent identification and confirmation of the compound of interest. This approach has been tested in this 182 study with two NPS. The common fragment approach is another interesting option for the detection of NPS. 183 Synthetic cannabinoids generally appear on the market with minor structural modifications which means that 
184 during fragmentation they lose the same specific functional groups and that structural information can be used

185 as a filter to detect those components. This tool has been tested with 14 different synthetic cannabinoids.

186 2.6 Quantification and method validation

187 The performance of the method was evaluated following EU guidelines with minor modifications[29, 30]. The

188 instrumental linearity of the method was studied by analyzing standard solution in triplicate at 9 different 189 concentrations that were between $0.25 \mathrm{ng} \mathrm{mL}^{-1}$ and $500 \mathrm{ng} \mathrm{mL}^{-1}$ (it would be equivalent to $1-2000 \mathrm{ng} \mathrm{L}^{-1}$ in 190 sewage after applying the pre-concentration factor). Satisfactory linearity was assumed when the correlation

191 factor $\left(\mathrm{R}^{2}\right)$ was $>0.99$, based on analyte/internal standard peak area ratio applied in different concentration 192 ranges for each compound. The most relevant aspects about the instrumental parameters are summarized in

193 Table S-2.

194 The theoretical limit of quantification $\left(\mathrm{LOQ}_{\mathrm{THE}}\right)$ for real samples was calculated based on the ratio between the 195 instrumental limit of quantification, estimated as a signal-to-noise $(\mathrm{S} / \mathrm{N})$ ratio of 10 for the standard in the 196 calibration curve at the lowest level, and the matrix suppression (MS) for each deuterated internal standard 197 multiplied by the concentration factor $(\mathrm{CF})$.

$$
\text { Theoretical } L O Q_{R S}=\frac{L O Q_{\text {inst }}}{M S} \times C F
$$

199 The matrix effect defined as Matuszewski et al.[35] was calculated as the ratio between the accurate mass signal of the 25 deuterated standard spiked in different sewage samples after extraction and the accurate mass

201 signal of the same deuterated standard in the calibration curve. The influence of the sewage matrix 202 concentration on the analysis is a crucial factor but simply controlled with a pre-concentration factor study[36].

203 For analytes where deuterated standards were unavailable, either the deuterated analyte with similar structure,

204 polarity or the closest retention time was selected for correction.

205 Precision was expressed as the repeatability of the method in terms of relative standard deviation and trueness

206 was tested by means of the absolute recovery due to the lack of quality control standards. The relative 207 recoveries and standard deviations of the whole procedure were calculated by analyzing sewage water samples 208 spiked at a low and high concentration with the analytes (100 and $\left.600 \mathrm{ng} \mathrm{L}^{-1}\right)$ according to the levels generally 209 found in wastewater and with $600 \mathrm{ng} \mathrm{L}^{-1}$ of the deuterated internal standard solution mix. All recovery 210 experiments were performed in six replicates $(n=6)$. Concentrations of target compounds found in wastewater 211 "blank" samples were subtracted from the spiked samples due to the absence of an appropriate wastewater 212 blank. Instrumental accuracy and precision was assessed by analyzing spiked Mili-Q water (n=5) at two 
213 different concentrations for each compound, low level (between points 2-3 on the calibration curve) and high

214 level (between points 8-9 on the calibration curve).

215 The stability of the target analytes in wastewater was evaluated in triplicate $(\mathrm{n}=3)$ via the analysis of $500 \mathrm{~mL}$ of

216 spiked (1000 $\left.\mathrm{ng} \mathrm{mL}^{-1}\right)$ wastewater immediately following spiking ( $\mathrm{t}=0$ hours) and again at $\mathrm{t}=5,8$ and 120 hours

217 post spiking. The wastewater sample ( $\mathrm{pH} 7.5)$ was stored in an amber glass bottle at $10{ }^{\circ} \mathrm{C}$ throughout the study

218 in order to best replicate the average wastewater influent temperature.

\section{RESULTS AND DISCUSSION}

2213.1 Sample extraction optimization

222 Selected parameters were investigated in order to optimize the method. Silanisation of the glassware, by

223 deactivating the ion-exchange groups with dichlorodimethylsilane, resulted in increased recoveries for some of

224 the more polar drugs, such as amphetamine-like compounds (Figure S-1). Elevated evaporation temperature 225 was also found to result in analyte losses, therefore special attention was paid to the drying of extracts .Figure

226 S-2 shows poor recoveries for some of the phenethylamines following nitrogen evaporation at $50^{\circ} \mathrm{C}$. All

227 samples were subsequently evaporated to around $100 \mu \mathrm{L}$ under a gentle nitrogen stream at $35^{\circ} \mathrm{C}$.

\section{$228 \quad 3.2$ Method performance}

229 The relative recoveries and standard deviations from the relative responses analyte/ILIS are shown in the Table

230 S-3. For the target analytes with corresponding ILIS the recoveries are typically $>80 \%$ with some exceptions

231 such as MDMA, norketamine and atomoxetine with slightly lower recoveries of around $70 \%$. The remaining

232 target analytes were corrected using the ILIS with the closest structure, polarity or eluting time, achieving 233 relative recoveries ranging from 60 to $118 \%$. However certain analytes such as clonazepam (56\%),

234 dehydronorketamine (53\%), methoxetamine (40\%) and methylphenidate (50\%) showed lower recoveries.

235 Relative recoveries at $100 \mathrm{ng} \mathrm{L}^{-1}$ are in general slightly lower due their proximity to the $\mathrm{LOQ}_{\mathrm{THE}}$, however in 236 general the recoveries do not substantially differ between the low and high concentration. The overall method 237 precision was calculated as the relative standard deviation (RSD) due to the lack of reference materials for the 238 wastewater samples, and showed satisfactory results $<20 \%$ RSD for all compounds.

239 Paracetamol and hydroxycotinine were detected at high concentrations in the blank (low ppb) making

240 subtraction and the recovery calculation not possible for paracetamol, and less precise for hydroxycotinine

241 (highest RSD). Carbamazepine also exhibits ionization issues in terms of signal enhancement with recoveries

242 slightly higher than $100 \%$ while other compounds with high signal suppression were gabapentin, $p$ - 
hydroxymethamphetamine, MDA, diclofenac, THC-OH and THC-COOH, which were finally excluded from

244 further validation in this study, but this does not however preclude the potential for lower matrix effects and

245 better analytical outcomes in wastewater from other locations or other sample types that present a cleaner

246 matrix.

247 The overall method recoveries were satisfactory and similar to those screening methods previously reported for

248 pharmaceuticals in wastewater samples [5]. Different target procedures, such as QqQ, with optimized and

249 specific conditions for each analyte, generally present greater sensitivity. This is a downside of all multi-

250 residue methods where the optimized conditions are compromised in order to accommodate several analytes,

251 especially in complex and variable matrix such as wastewater. Nevertheless the good sensitivity levels and

252 dynamic range presented in the new HR-MS instruments together with the instrumental performance in terms

253 of repeatability and sensitivity helps to counteract low recoveries.

254 3.3 Quantification and method validation

255 Table S-2 provides an overview of the performance of the developed target method. The instrumental linearity

256 for nine concentration levels is in the range of 0.25 to $400 \mathrm{ng} \mathrm{mL}^{-1}$, equivalent to $1-2,000 \mathrm{ng} \mathrm{L}^{-1}$ in wastewater

257 after applying the pre-concentration factor. These are within the levels reported in literature[16] showing good

258 results for all of the initial 51 selected compounds presenting correlation coefficients greater than 0.99.

259 Different ranges were applied for every compound according to sensitivity. Instrumental LOQ was calculated

260 as the signal-to-noise $(S / N)$ ratio of 10 for the standard in the calibration curve at the lowest concentration.

261 Instrumental accuracy and precision, both for intra- and inter-day, was assessed at two different concentration

262 levels for each compound showing an satisfactory accuracy levels between 87.8-113.1\% range and precision

$263<12.8 \%(\mathrm{RSD})$.

264 The theoretical limits of quantification (LOQ ${ }_{\mathrm{THE}}$, Table S-3), calculated based on the ratio between the 265 instrumental limit of quantification and matrix suppression multiplied by the concentration factor for each

266 deuterated internal standard, varied between 0.4 and $187 \mathrm{ng} \mathrm{\textrm {L } ^ { - 1 }}$. This is just a valuable estimation about the 267 method performance and due to the complexity of the matrix common sense has to be used prior the analysis of

268 a new batch of samples.

269 The instrumental performance was also evaluated in terms of the intra-day (repeatability) and inter-day 270 (reproducibility) precision studies from five spiked Mili-Q water samples at two different concentration levels 271 in five repeated injections and three consecutive days. The results in terms of RSD show values below $10 \%$ in 272 both intra and inter-day studies for all the compounds except for THC-COOH at the low concentration level for 273 the intra-day (10.2\%) and amphetamine and flunitrazepam at the low concentration level for the inter-day (11.8 

mass error for all the target compounds (mass error range between - 0.51 and $0.39 \mathrm{mDa}$, (Table S-2) ensure the reliability of the instrument performance, an essential aspect in HRMS.

\section{3.4 Liquid Chromatography-Mass Spectrometry}

278 Suppression or enhancement of the target ion signal in LC-MS is a critical issue in wastewater analysis due to the complex nature of the sample and the co-elution of the analytes with matrix constituents. The negative

280 consequences of this problem are best avoided with the use of appropriate ILIS, together with extensive sample 281 pre-treatment (clean-up), chromatographic separation and sample dilution. The effects of the sample matrix was evaluated by comparing the matrix effect/pre-concentration factor ratio for all the 25 deuterated internal standards. Deuterated internal standards are not present in sewage water samples but are affected by the same

284 potential losses during the analytical process as the analyte of interest. The influence of the SPE loading volume on matrix suppression was optimized by spiking $100 \mathrm{ng}$ of the deuterated internal standard mix solution into the extracts of 3 different wastewater extracts at 4 different volumes (100, 200, 300, and $400 \mathrm{~mL}$ ).

287 The accurate mass signal was compared with the accurate mass signal of the deuterated internal standards in solvent. A loading volume of $100 \mathrm{~mL}$ was found to give the most satisfactory compromise for the analysis with a pre-concentration factor of 250 (LC vial volume $400 \mu \mathrm{L}$, injection volume $5 \mu \mathrm{L}$ ) (Figure S-3). Van Nuijs et al.[37]considered not including matrix effect studies in method validation, claiming that matrix

291 effects can be highly variable between samples. We agree on the fact that each individual sample has a 292 different composition, but we strongly recommend the matrix characterization of different batches of wastewater samples when they come from different locations or affected by different conditions since an

294 appropriate sample dilution can improve substantially the general performance of the method. Generally 295 analytical methods rely on the validated LOQ but wastewater analysis required a more intense day-to-day 296 control.

\subsection{Stability in wastewater}

298 The stability of the analytes in wastewater was investigated to ensure the use of appropriate sample-storage and

299 handling procedures. The study was configured to estimate the degradation of our target compounds during the 300 in-pipe transit time for a single wastewater sample.

301 Table S-4 shows the results for the stability test for the target analyte signal in non-filtered wastewater at $\mathrm{pH}$

3027.5 and $10^{\circ} \mathrm{C}$ expressed as the difference between initial and final concentrations at different time points $(\mathrm{t}=0$,

3035,8 and 120 hours). Time points were set according the mean residence time in the sewer system from Oslo to 
304 the VEAS WWTP, 5 hours, the total treatment time in the plant, 3 hours, and finally a long exposure during

305120 hours to provide an estimation of the biodegradation after 5 days.

306 Over the first 5 hours EDDP is the only analyte with major change in peak area (45\% higher). Between 5 and 8

307 hours significant changes were also observed for hydroxycotinine, benzoylecgonine, methylphenidate the 308 concentration changed substantially $(> \pm 30 \%)$. The results following 120 hours show degradation for the many

309 of the target analytes, such as morphine (83\%), $p$-hydroxymethamphetamine (70\%), paracetamol (-88\%), MDA

$310(-96 \%)$, methylphenidate (-88\%), cocaine (-75\%), EDDP (77\%), nitrazepam (-79\%) and phenazepam (-70\%).

311 These data provide an indication of the transformation that may occur during in-sewer transport. The results are

312 in accordance with previous reports [38].

313 3.6 Application to sewage water samples

314 3.6.1 Target Screening.

315 The main objective of this work was to demonstrate the broad applicability of a multi-residue method in MS

316 by UHPLC-QTOF for both screening and quantification purposes. The validated method was applied to the

317 analysis of 15 wastewater samples collected from two WWTPs in Oslo and Trondheim in 2014 to assess the

318 applicability of the method. Table 2 shows the population normalized loads for the target drugs in wastewater,

319 estimated from the daily concentration of each detected target compound in the wastewater sample, daily flow

320 discharged into the WWTP and by the population size (mg day ${ }^{-1} 1000$ inhabitants $\left.{ }^{-1}\right)$. Results from Oslo show

321 the daily average loads calculated from the analysis in triplicate of a daily composite sample taken every

322 Friday, Saturday and Sunday during three consecutive weekends.

323 In general, pharmaceuticals were detected in higher concentration for both cities. Atenolol, paracetamol,

324 metoprolol, propranolol, citalopram, carbamazepine, oxazepam were detected at elevated concentrations in all

325 of the samples from Oslo and Trondheim. Methylphenidate and alprazolam were only present in samples from

326 Trondheim, suggesting different levels of consumption within the same country.

327 The heroin metabolites, 6-MAM and morphine, were detected in samples from both locations but it is 328 important to remember that while 6-MAM is specific to heroin, the presence of morphine in wastewater also 329 can result from the clinical use of legal pharmaceuticals containing morphine or codeine. Methadone and its 330 metabolite, EDDP, are usually present in wastewater samples and in this study the loads obtained in Oslo are 331 approximately ten times higher than those from Trondheim. Biomarkers of other commonly used drugs, such as 332 cocaine, benzoylecgonine, amphetamine and methamphetamine were detected in samples from both cities at 333 different concentrations. While the loads of cocaine/benzoylecgonine are approximately ten timers higher in

334 Oslo than in Trondheim, the results for amphetamine and methamphetamine are slightly higher in Trondheim 
335 suggesting different use patterns. This is in agreement with the general geographical distribution of the drugs in

336 Norway published in the Annual report to the European Monitoring Centre for Drugs and Drug Addition[39]

337 where cocaine seems to be a big city phenomenon with special relevance in Oslo and at the same time within

338 the European context Norway has been shown to have one of the biggest market for 339 amphetamine/methamphetamine[39].

340 Cocaethylene, the main urinary biomarker of cocaine co-consumption with alcohol, and MDMA were

341 identified and quantified only in Oslo. Methylone, ketamine and methoxetamine were found in at least one of

342 the composite samples from Oslo suggesting a sporadic weekend use.

343 This method is also been successfully used for the analysis of other sewage-based samples, such as pooled

344 urine samples and passive sampler extracts. Sample preparation was adapted for each case (SI). It is worth

345 mentioning the importance of the dilution factor for both POCIS and pooled urine samples since the

346 concentration level for the target biomarkers are extraordinary high compared with those typically found in

347 wastewater, being a very suitable samples for suspect screenings.

348 Table S-5 and S-6 show the results of the target analysis performed on 10 POCIS extracts from the winter of

3492013 and three pooled urine samples collected from three different music festivals in summer of 2014.The

350 results for the passive sampling devices must be corrected with an exposure factor, however this is outside of

351 the scope of this paper and therefore the results are presented as amount of analyte (ng) per POCIS. The target

352 method was also used for the analysis of the pooled urine samples collected from music festivals.

353 In general the compounds identified in the POCIS do not differ with the compounds obtained and reported in

354 this study for the wastewater samples, however in the pooled urine samples the differences are greater,

355 especially for the amphetamine-like compounds exposed to a lower dilution factor compared with the other

356 sewage-based samples. The low dilution factor makes pooled urine samples a very suitable sample for suspect

357 screening purposes.

$358 \quad$ 3.6.2 Suspect Screening

359 The suspect screening database was applied to the pooled urine samples to demonstrate the applicability of 360 these libraries. The aim of this work was to develop a reiterative tool to track the presence of the target 361 compounds in wastewater by complementing the 51 target analytes with new targets identified via suspect

362 screening The 51 compounds were selected as described above, however as shown in Table 2 not all were 363 present or detected in the sewage-based samples. Suspect screening together with retrospective analysis allows 364 the selection of new compounds, based on detection in the wastewater to be added to the quantitative target 
method through the use of an authentic reference standard thereby facilitating decision-making and improving cost effectiveness.

367 The continuum data for the three-music festival samples were peak detected using the processing settings described above. Following peak processing UNIFI provided a list of $\mathrm{m} / \mathrm{z}$ values termed as candidates. The number of candidates for the samples were $11,220,11,183$ and 11,164 for each festival. The acceptance criterion for the tentative candidates is within the tolerance range described in the data processing section. Despite this, in this method the authors defined limit checks broader to perform the final step manually, meaning that after the evaluation of the candidates against the database UNIFI provided a new list with 182,

373151 and 131 tentative IDs respectively. Table S-7 shows some examples of positive identifications for the ID's

374 fulfilling the criteria consisted of a mass accuracy $\pm 5 \mathrm{ppm}$, retention time $\pm 0.5 \mathrm{~min}$, isotope match $\pm 5 \mathrm{ppm}$ and

375 a minimum of two detected ions in the HE. Sildenafil, a medicine to treat erectile dysfunction, zolpidem,

376 prescription medication for the treatment of insomnia, and benzocaine and lidocaine, known cocaine cutting

377 agents are interesting for future target analysis. In this case the 24 compounds identified (Table S-7) would move from the level 3 (tentative candidates) to the level 2 (probable structure) in the absence of the reference standards for the final confirmation [33].

In order to augment the suspect database used in this study, a non-target approach was used to net purchased

381 NPS; 5/6-APB and N-adamantyl-1-pentylindole-3-carboxamide (commercial names: Benzo Fury and 2NE1-

382 APICA). Figures S-4 and S-5 illustrate the detection and identification of the 5/6-APB and N-adamantyl-1pentylindole-3-carboxamide. UNIFI automatically detects the sample components, and subsequently through the accurate mass spectra allows the identification of the unknown candidates. In contrast with the characterization analysis of the classic drugs, the NPS chromatograms are relatively clean since they are purchased in almost pure form without any additive or "cutting agent" as is shown in Figure $\mathbf{S - 4}$ and $\mathbf{S - 5}$ where only two peaks are present as the most abundant. As an example, Figure S-5 shows a chromatogram with the assigned fragments (159.0798 and 131.0488) from the accurate mass ion 176.1065 at $5.2 \mathrm{~min}$.

389 A common fragment approach was used also used as a complementary tool for the identification and subsequently introduction of 14 synthetic cannabinoids into the suspect database. Figure S-6 shows the total ion chromatogram of the 14 synthetic cannabinoids described in the picture together with the chromatogram of the common fragment $\mathrm{m} / \mathrm{z} 155.0492$ belonging to 8 of the synthetic cannabinoids. 


\section{CONCLUSIONS}

397 A high confidence multi-residue method using auto-SPE-UHPLC-QTOF for the determination of 51 drugs,

398 pharmaceuticals and metabolites was validated and applied to different sewage-base samples. Meanwhile, this analytical procedure is also intended to fulfill the analytical challenge of the detection and reliable

400 identification of NPS appearing in the market through the application of a suspect screening approach

401 augmented by a reiterative process intended to both update the target multi-residue method, and the suspect 402 screening list.

403 The selection of HLB as an extraction sorbent and C18 as the chromatography column provides a broad and generic methodology for the rapid identification of a larger number of compounds dealing with the compromise

405 between elimination of interfering matrix components (using more selective extraction procedures) or the

406 unification of all requested features in just one method. The method has a satisfactory linear dynamic range and

407 the sensitivity. Even though many papers have shown the capability of the HRMS screening techniques just for

408 qualitative purposes, the high selectivity, accurate mass and the information provided by the fragments as mass

409 defect or the isotopic pattern, make this method in a very reliable tool also for the quantification of drugs and 410 emergent substances in wastewater.

411 The described reiterative and dynamic workflow has been designed in order to constantly update the screening

412 levels based on experimental data (Figure 3 Target analysis can be supplemented by simultaneously screening

413 through the suspect database in order to identify new compound of interest, all in one injection. If a suspect

414 candidate is identified, this candidate can be later confirmed by purchasing the reference standard and this

415 compound can then be incorporated into the target method. Despite not being used in this work, non-target

416 screening is an additional avenue of the described workflow which can enhance the potential of the approach.

417 The application of this method was finally tested with different real samples showing an extensive list of

418 confirmed compounds in different sewage-based samples confirming the broad applicability of the developed

419 method. Two NPS were also introduced into the suspect database together with another 14 synthetic

420 cannabinoids as part of the strategy to expand the library for further identification purposes in the future.

\section{ACKNOWLEDGEMENTS}

423 The authors would like to thank Pia Ryrfors, VEAS WWTP, for providing access to the sampling area and the 424 best information regarding the wastewater samples. Jose Antonio Baz Lomba acknowledges the EU 425 International Training Network SEWPROF (Marie Curie-FP7-PEOPLE, grant number 317205) for his Early 426 Stage Researcher grant 
430 [1] UNODC, World Drug Report 2014, United Nations Office on Drugs and Crime 431 (UNODC), 2014.

432 [2] EMCDDA, European Drug Report 2015: Trends and Developments, European 433 Monitoring Centre for Drugs and Drug Addiction, Lisbon, 2015.

434 [3] EMCDDA, New psychoactive substances in Europe. An update from the EU Early

435 Warning System, in: P.O.o.t.E. Union (Ed.), European Monitoring Centre for Drugs and 436 Drug Addiction, Luxembourg, 2015.

437 [4] L. Bijlsma, J.V. Sancho, F. Hernandez, W.M. Niessen, Fragmentation pathways of 438 drugs of abuse and their metabolites based on QTOF MS/MS and MS(E) accurate-mass 439 spectra, Journal of mass spectrometry : JMS, 46 (2011) 865-875.

440 [5] M. Gros, S. Rodriguez-Mozaz, D. Barcelo, Fast and comprehensive multi-residue 441 analysis of a broad range of human and veterinary pharmaceuticals and some of their 442 metabolites in surface and treated waters by ultra-high-performance liquid 443 chromatography coupled to quadrupole-linear ion trap tandem mass spectrometry, $\mathrm{J}$ 444 Chromatogr A, 1248 (2012) 104-121.

445 [6] C. Ort, M.G. Lawrence, J. Reungoat, G. Eaglesham, S. Carter, J. Keller,

446 Determining the fraction of pharmaceutical residues in wastewater originating from a 447 hospital, Water Research, 44 (2010) 605-615.

448 [7] K.V. Thomas, C. Dye, M. Schlabach, K.H. Langford, Source to sink tracking of 449 selected human pharmaceuticals from two Oslo city hospitals and a wastewater 450 treatment works, J Environ Monit, 9 (2007) 1410-1418.

451 [8] K. Langford, K.V. Thomas, Input of selected human pharmaceutical metabolites into the Norwegian aquatic environment, Journal of Environmental Monitoring, 13 (2011) 416-421.

454 [9] T.L. ter Laak, P.J. Kooij, H. Tolkamp, J. Hofman, Different compositions of pharmaceuticals in Dutch and Belgian rivers explained by consumption patterns and treatment efficiency, Environ Sci Pollut Res Int, 21 (2014) 12843-12855. [10] D.R. Baker, B. Kasprzyk-Hordern, Spatial and temporal occurrence of pharmaceuticals and illicit drugs in the aqueous environment and during wastewater treatment: New developments, Science of The Total Environment, 454-455 (2013) 442456.

[11] P. Verlicchi, M. Al Aukidy, A. Jelic, M. Petrović, D. Barceló, Comparison of measured and predicted concentrations of selected pharmaceuticals in wastewater and surface water: A case study of a catchment area in the Po Valley (Italy), Science of The Total Environment, 470-471 (2014) 844-854.

465 [12] A.L. van Nuijs, A. Covaci, H. Beyers, L. Bervoets, R. Blust, G. Verpooten, H.

466 Neels, P.G. Jorens, Do concentrations of pharmaceuticals in sewage reflect prescription 467 figures?, Environ Sci Pollut Res Int, 22 (2015) 9110-9118.

468 [13] K.V. Thomas, M.J. Reid, What Else Can the Analysis of Sewage for Urinary 469 Biomarkers Reveal About Communities?, Environmental Science \& Technology, 45 $470 \quad$ (2011) 7611-7612.

471 [14] EMCDDA, Wastewater analysis and drugs: a European multi-city study, European 472 Monitoring Centre for Drugs and Drug Addiction, 2015. 
[15] S. Castiglioni, K.V. Thomas, B. Kasprzyk-Hordern, L. Vandam, P. Griffiths,

474 Testing wastewater to detect illicit drugs: state of the art, potential and research needs,

475 The Science of the total environment, 487 (2014) 613-620.

476 [16] A.L.N. van Nuijs, S. Castiglioni, I. Tarcomnicu, C. Postigo, M.L. de Alda, H.

477 Neels, E. Zuccato, D. Barcelo, A. Covaci, Illicit drug consumption estimations derived

478 from wastewater analysis: A critical review, Science of The Total Environment, 409

479 (2011) 3564-3577.

480 [17] C. Harman, M. Reid, K.V. Thomas, In situ calibration of a passive sampling device

481 for selected illicit drugs and their metabolites in wastewater, and subsequent year-long

482 assessment of community drug usage, Environ Sci Technol, 45 (2011) 5676-5682.

483 [18] I. Gonzalez-Marino, J.B. Quintana, I. Rodriguez, M. Gonzalez-Diez, R. Cela,

484 Screening and selective quantification of illicit drugs in wastewater by mixed-mode

485 solid-phase extraction and quadrupole-time-of-flight liquid chromatography-mass

486 spectrometry, Analytical chemistry, 84 (2012) 1708-1717.

487 [19] K.V. Thomas, L. Bijlsma, S. Castiglioni, A. Covaci, E. Emke, R. Grabic, F.

488 Hernandez, S. Karolak, B. Kasprzyk-Hordern, R.H. Lindberg, M. Lopez de Alda, A.

489 Meierjohann, C. Ort, Y. Pico, J.B. Quintana, M. Reid, J. Rieckermann, S. Terzic, A.L.

490 van Nuijs, P. de Voogt, Comparing illicit drug use in 19 European cities through sewage

491 analysis, The Science of the total environment, 432 (2012) 432-439.

492 [20] C. Ort, A.L. van Nuijs, J.D. Berset, L. Bijlsma, S. Castiglioni, A. Covaci, P. de

493 Voogt, E. Emke, D. Fatta-Kassinos, P. Griffiths, F. Hernandez, I. Gonzalez-Marino, R.

494 Grabic, B. Kasprzyk-Hordern, N. Mastroianni, A. Meierjohann, T. Nefau, M. Ostman,

495 Y. Pico, I. Racamonde, M. Reid, J. Slobodnik, S. Terzic, N. Thomaidis, K.V. Thomas,

496 Spatial differences and temporal changes in illicit drug use in Europe quantified by

497 wastewater analysis, Addiction, 109 (2014) 1338-1352.

498 [21] E. Zuccato, S. Castiglioni, M. Tettamanti, R. Olandese, R. Bagnati, M. Melis, R.

499 Fanelli, Changes in illicit drug consumption patterns in 2009 detected by wastewater

500 analysis, Drug Alcohol Depend, 118 (2011) 464-469.

501 [22] F.Y. Lai, R. Bruno, W. Hall, C. Gartner, C. Ort, P. Kirkbride, J. Prichard, P.K.

502 Thai, S. Carter, J.F. Mueller, Profiles of illicit drug use during annual key holiday and

503 control periods in Australia: wastewater analysis in an urban, a semi-rural and a

504 vacation area, Addiction, 108 (2013) 556-565.

505 [23] J.R. Archer, P.I. Dargan, H.M. Lee, S. Hudson, D.M. Wood, Trend analysis of

506 anonymised pooled urine from portable street urinals in central London identifies

507 variation in the use of novel psychoactive substances, Clin Toxicol (Phila), 52 (2014)

508 160-165.

509 [24] M.J. Reid, L. Derry, K.V. Thomas, Analysis of new classes of recreational drugs in

510 sewage: synthetic cannabinoids and amphetamine-like substances, Drug Test Anal, 6

511 (2014) 72-79.

512 [25] J. Kinyua, A. Covaci, W. Maho, A.K. McCall, H. Neels, A.L. van Nuijs, Sewage-

513 based epidemiology in monitoring the use of new psychoactive substances: Validation

514 and application of an analytical method using LC-MS/MS, Drug Test Anal, (2015) n/a-

$515 \mathrm{n} / \mathrm{a}$.

516 [26] M. Ibáñez, J.V. Sancho, L. Bijlsma, A.L.N. van Nuijs, A. Covaci, F. Hernández,

517 Comprehensive analytical strategies based on high-resolution time-of-flight mass

518 spectrometry to identify new psychoactive substances, TrAC Trends in Analytical

519 Chemistry, 57 (2014) 107-117. 
[27] F. Hernández, M. Ibáñez, R. Bade, L. Bijlsma, J.V. Sancho, Investigation of pharmaceuticals and illicit drugs in waters by liquid chromatography-high-resolution mass spectrometry, TrAC Trends in Analytical Chemistry, 63 (2014) 140-157. [28] M. Ibanez, L. Bijlsma, A.L. van Nuijs, J.V. Sancho, G. Haro, A. Covaci, F. Hernandez, Quadrupole-time-of-flight mass spectrometry screening for synthetic cannabinoids in herbal blends, Journal of mass spectrometry : JMS, 48 (2013) 685-694. [29] U.Ö. B. Magnusson, Eurachem Guide: The Fitness for Purpose of Analytical Methods - A Laboratory Guide to Method Validation and Related Topics, ISBN 97891-87461-59-0 (2nd ed. 2014). [30] Commission Decision 2002/657/EC of 12 August 2002implementing Council Directive 96/23/EC concerning to theperformance of analytical methods and the implementationof the results, Official Journal of the European Communities, (2002) 8 62.

[31] R. Lee, M. Roberts, A. Paccou, M. Wood, Development of a new UPLC/MS method for systematic toxicological analysis, Waters Corp. Application Note 72002905EN, (2009).

[32] M. Krauss, H. Singer, J. Hollender, LC-high resolution MS in environmental analysis: from target screening to the identification of unknowns, Analytical and bioanalytical chemistry, 397 (2010) 943-951.

[33] E. Schymanski, H. Singer, J. Slobodnik, I. Ipolyi, P. Oswald, M. Krauss, T. Schulze, P. Haglund, T. Letzel, S. Grosse, N. Thomaidis, A. Bletsou, C. Zwiener, M. Ibáñez, T. Portolés, R. de Boer, M. Reid, M. Onghena, U. Kunkel, W. Schulz, A. Guillon, N. Noyon, G. Leroy, P. Bados, S. Bogialli, D. Stipaničev, P. Rostkowski, J. Hollender, Non-target screening with high-resolution mass spectrometry: critical review using a collaborative trial on water analysis, Analytical and bioanalytical chemistry, (2015) 1-19.

[34] M.J. Reid, J.A. Baz-Lomba, Y. Ryu, K.V. Thomas, Using biomarkers in wastewater to monitor community drug use: A conceptual approach for dealing with new psychoactive substances, Science of the Total Environment, 487 (2014) 651-658. [35] B.K. Matuszewski, M.L. Constanzer, C.M. Chavez-Eng, Strategies for the assessment of matrix effect in quantitative bioanalytical methods based on HPLCMS/MS, Analytical chemistry, 75 (2003) 3019-3030.

[36] L. Bijlsma, E. Beltrán, C. Boix, J.V. Sancho, F. Hernández, Improvements in analytical methodology for the determination of frequently consumed illicit drugs in urban wastewater, Analytical and bioanalytical chemistry, (2014) 1-12. [37] A.L. van Nuijs, A. Gheorghe, P.G. Jorens, K. Maudens, H. Neels, A. Covaci, Optimization, validation, and the application of liquid chromatography-tandem mass spectrometry for the analysis of new drugs of abuse in wastewater, Drug Test Anal, 6 (2014) 861-867.

[38] S. Castiglioni, L. Bijlsma, A. Covaci, E. Emke, F. Hernandez, M. Reid, C. Ort, K.V. Thomas, A.L. van Nuijs, P. de Voogt, E. Zuccato, Evaluation of uncertainties associated with the determination of community drug use through the measurement of sewage drug biomarkers, Environ Sci Technol, 47 (2013) 1452-1460.

[39] The Drug Situation in Norway. Annual report to the European Monitoring Centre for Drugs and Drug Addiction - EMCDDA Norwegian Institute for Alcohol and Drug Research (SIRUS), Oslo, 2015. 
568 Figures and Tables

569

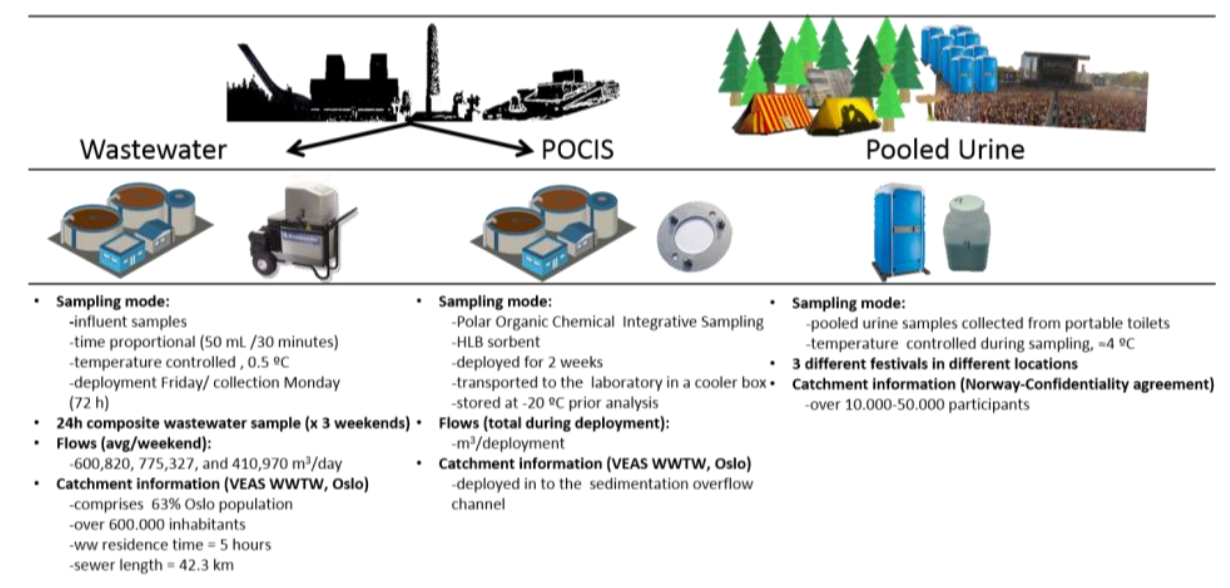

570

Figure1

572 Different sewage-based samples used in this study

573

574

575

Page 18 of 22 


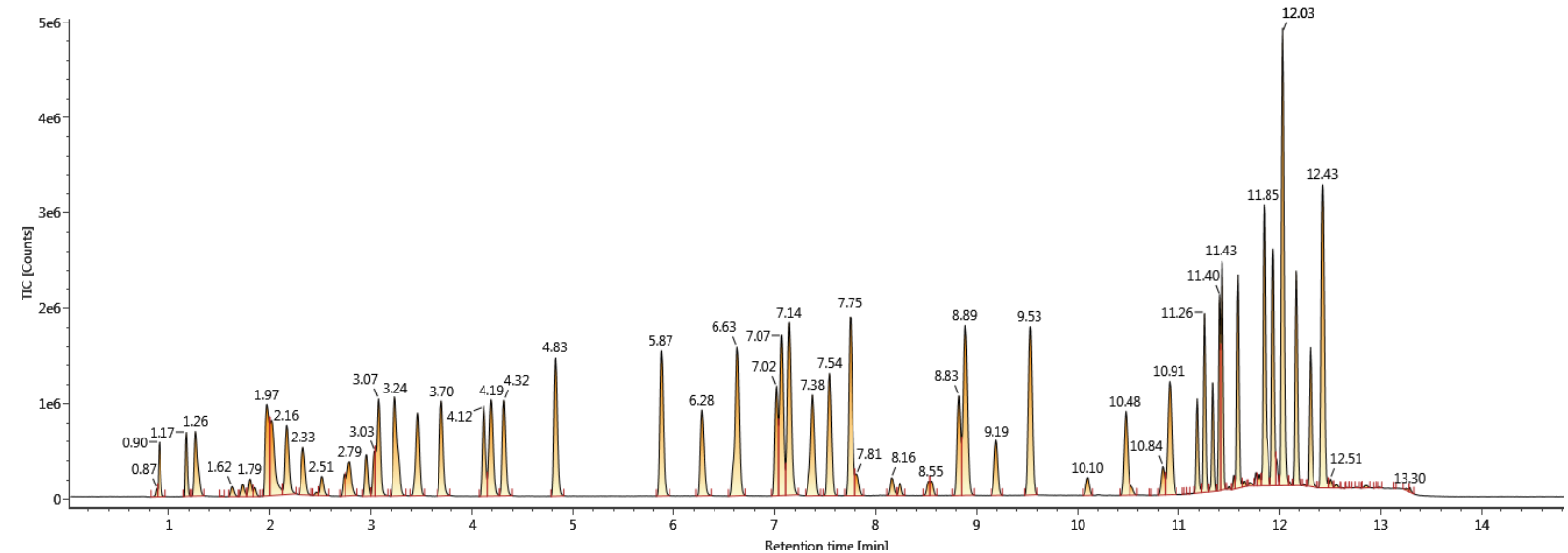

$578 \quad$ Figure 2

579 2D mass chromatogram of the low energy channel (6eV ESI+) of a $100 \mathrm{ng} / \mathrm{mL}$ standard mix solution with all 580 the target compounds, UNIFI (Waters Corporation, Milford MA, USA)

581

582 


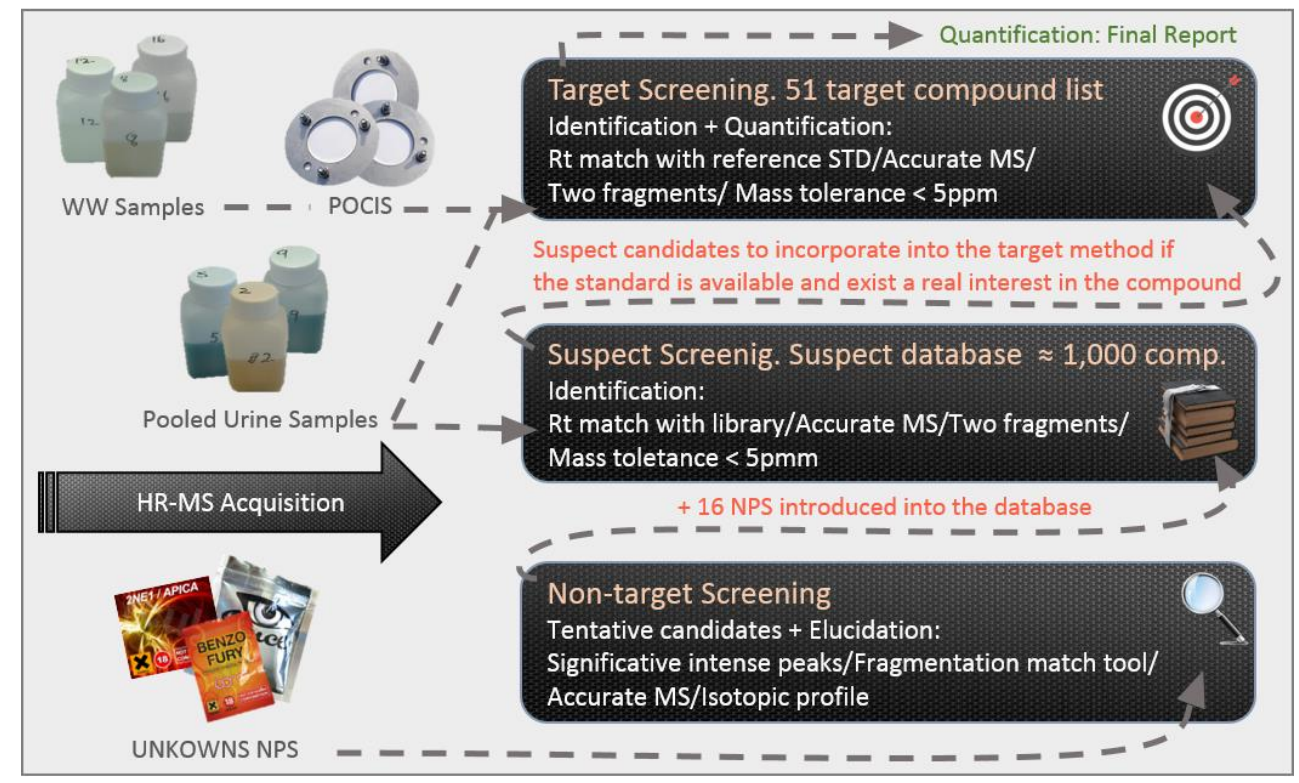

585 Figure 3. Illustrative workflow diagram for the three different approaches developed in this study.

586

587 
Table 1

590 Target compounds organized by their retention times with the experimental parameters.

\begin{tabular}{|c|c|c|c|c|c|}
\hline \multirow[t]{2}{*}{ Compound } & \multirow[t]{2}{*}{ Internal Standard } & \multirow[t]{2}{*}{$t_{R}$} & \multirow{2}{*}{$\begin{array}{c}\text { Precursor ion } \\
{[M+H]+}\end{array}$} & \multirow{2}{*}{$\begin{array}{c}\text { Product ion } \\
(\mathrm{m} / \mathrm{z})\end{array}$} & \multirow{2}{*}{$\begin{array}{r}\text { Linear rang } \\
\left(n g m L^{-1}\right)\end{array}$} \\
\hline & & & & & \\
\hline Morphine & Atenolol-d 7 & 1.17 & 286.14376 & 201.0910 & $0,5-100$ \\
\hline Hydroxycotinine & Atenolol-d 7 & 1.17 & 193.09714 & 134.0595 & $0,5-400$ \\
\hline Atenolol & Atenolol-d 7 & 1.26 & 267.1703 & 190.0863 & $0,5-200$ \\
\hline Salbutamol & Atenolol-d 7 & 1.28 & 240.1594 & 145.0648 & $1-100$ \\
\hline p-Hydroxymethamphetamine & Atenolol-d 7 & 1.31 & 166.12263 & 135.0798 & $2-100$ \\
\hline Paracetamol & Atenolol-dr & 1.62 & 152.07059 & 110.0601 & $2-400$ \\
\hline Cathinone & Methcathinone- $\mathrm{d}_{3}$ & 1.75 & 150.09133 & 132.0808 & $5-400$ \\
\hline Gabapentin & Gabapentin- $\mathrm{d}_{10}$ & 1.8 & 172.13319 & 154.1227 & $5-200$ \\
\hline Pseudoephedrine & Methcathinone- $\mathrm{d}_{3}$ & 1.96 & 166.12263 & 133.0886 & $5-400$ \\
\hline Methcathinone (Ephedrone) & Methcathinone- $\mathrm{d}_{3}$ & 1.98 & 164.10698 & 146.0971 & $1-400$ \\
\hline Methylone & Methylone- $\mathrm{d}_{3}$ & 2.16 & 208.09681 & 160.0757 & $0,25-400$ \\
\hline 6-MAM & Methylone- $\mathrm{d}_{3}$ & 2.29 & 328.15432 & 165.0699 & $0,5-400$ \\
\hline Amphetamine & Amphetamine-ds & 2.41 & 136.11207 & 91.0543 & $5-400$ \\
\hline MDA & MDA-d5 & 2.47 & 180.10189 & 163.0754 & $10-200$ \\
\hline PMA & MDMA-ds & 2.53 & 166.12263 & 91.0543 & $2-400$ \\
\hline Methamphetamine & Methamphetamine-ds & 2.72 & 150.12771 & 119.0856 & $2-200$ \\
\hline MDMA & MDMA-ds & 2.75 & 194.11754 & 163.0754 & $2-400$ \\
\hline Dehydronorketamine & Norketamine- $\mathrm{d}_{4}$ & 2.89 & 222.06801 & 205.0415 & $1-400$ \\
\hline PMMA & MDMA-d5 & 2.98 & 180.13828 & 121.0648 & $2-400$ \\
\hline Benzoylecgonine & Benzoylecgonine- $\mathrm{d}_{3}$ & 3.07 & 290.13867 & 168.1020 & $0,25-400$ \\
\hline Mephedrone (4-MMC) & Mephedrone- $\mathrm{d}_{3}$ & 3.19 & 178.12263 & 160.1127 & $2-400$ \\
\hline Norketamine & Norketamine- $\mathrm{d}_{4}$ & 3.21 & 224.08366 & 125.0153 & $5-200$ \\
\hline Ketamine & Ketamine- $\mathrm{d}_{4}$ & 3.45 & 238.09931 & 125.0153 & $0,5-400$ \\
\hline 4-MEC & Ketamine- $d_{4}$ & 3.7 & 192.13828 & 174.1278 & $0,5-400$ \\
\hline Methoxetamine & Metoprolol-d $\mathrm{d} 7$ & 4.12 & 248.16449 & 121.0648 & $0,25-400$ \\
\hline Metoprolol & Metoprolol-d $\mathrm{d} 7$ & 4.19 & 268.1907 & 116.1070 & $0,25-400$ \\
\hline Methylphenidate & Metoprolol-d $\mathrm{d} 7$ & 4.32 & 234.14884 & 84.0808 & $0,25-400$ \\
\hline Cocaine & Cocaine-d3 & 4.83 & 304.15432 & 182.1176 & $0,25-200$ \\
\hline Cocaethylene & Cocaine-d3 & 5.87 & 318.16997 & 196.1333 & $0,25-400$ \\
\hline Propranolol & Fentanyl-ds & 6.28 & 260.16449 & 116.1070 & $0,25-200$ \\
\hline Fentanyl & Fentanyl-ds & 6.63 & 337.22742 & 188.1434 & $0,25-400$ \\
\hline AH-7921 & Fentanyl-d5 & 7.02 & 329.11818 & 284.0611 & $0,25-400$ \\
\hline Citalopram & Buprenorphine- $\mathrm{d}_{4}$ & 7.07 & 325.17105 & 109.0449 & $0,25-200$ \\
\hline Midazolam & Buprenorphine-dt & 7.14 & 326.08547 & 291.1167 & $0,25-400$ \\
\hline Buprenorphine & Buprenorphine-d4 & 7.38 & 468.31081 & 414.2633 & $0,25-400$ \\
\hline Carbamazepine & Buprenorphine-d4 & 7.54 & 237.10223 & 194.0965 & $0,25-200$ \\
\hline EDDP & Atomoxetine- $\mathrm{d} 7$ & 7.75 & 278.19031 & 234.1277 & $0,25-400$ \\
\hline Atomoxetine & Atomoxetine- $d 7$ & 7.81 & 256.16958 & 148.1103 & $1-200$ \\
\hline Nitrazepam & Oxazepam-d5 & 8.16 & 282.08731 & 236.0945 & $1-400$ \\
\hline Oxazepam & Oxazepam-d5 & 8.22 & 287.05817 & 241.0528 & $2-400$ \\
\hline Clonazepam & Oxazepam-d5 & 8.5 & 316.04833 & 270.0555 & $1-400$ \\
\hline Lorazepam & Oxazepam-d5 & 8.6 & 321.0192 & 275.0138 & $5-400$ \\
\hline Alprazolam & Oxazepam-d5 & 8.83 & 309.09014 & 281.0715 & $0,25-200$ \\
\hline Methadone & Methadone- $\mathrm{d}_{3}$ & 8.89 & 310.21652 & 265.1587 & $0,25-400$ \\
\hline Flunitrazepam & Diazepam-d5 & 9.19 & 314.09353 & 268.1007 & $1-400$ \\
\hline Etizolam & Diazepam-d5 & 9.53 & 343.07786 & 314.0391 & $0,25-200$ \\
\hline Phenazepam & Diazepam-d5 & 10.1 & 348.97382 & 196.1706 & $2-400$ \\
\hline Diazepam & Diazepam-ds & 10.91 & 285.07891 & 193.0886 & $0,25-200$ \\
\hline Diclofenac & Diazepam-ds & 11.4 & 296.02395 & 215.0496 & $5-400$ \\
\hline THC-OH & THC-OH-d 3 & 11.83 & 331.22675 & 313.2162 & $5-400$ \\
\hline THC-COOH & THC-COOH- $\mathrm{d}_{3}$ & 11.85 & 345.20602 & 299.2009 & $5-400$ \\
\hline
\end{tabular}


Table 2

594 Loads (mg day-1 1000 inhabitants-1) of detected analytes in influent wastewater samples from the Oslo (mean $595 \pm$ SD) and Trondheim (mean, n=1) WWTPs

a founded it at least one of the daily samples (not presented in all)

\begin{tabular}{|c|c|c|c|c|c|c|c|c|c|c|c|c|}
\hline \multirow{3}{*}{$\begin{array}{l}\text { Analyte } \\
\text { Morphine }\end{array}$} & \multicolumn{6}{|c|}{ Oslo, February 2014} & \multicolumn{6}{|c|}{ Trondheim, Spring 2014} \\
\hline & \multicolumn{2}{|c|}{ 1st weekend } & \multicolumn{2}{|c|}{ 2nd weekend } & \multicolumn{2}{|c|}{ 3rd weekend } & \multirow{2}{*}{$\begin{array}{c}\begin{array}{c}\text { Friday } \\
\text { 28.02.14 }\end{array} \\
6.1\end{array}$} & $\begin{array}{c}\text { Saturday } \\
\text { o8.03.14 }\end{array}$ & \multirow{2}{*}{$\begin{array}{c}\begin{array}{c}\text { Friday } \\
21.03 .14\end{array} \\
8.7\end{array}$} & \multirow{2}{*}{$\begin{array}{c}\begin{array}{r}\text { Saturday } \\
29.03 .14\end{array} \\
5.5\end{array}$} & \multirow{2}{*}{$\begin{array}{c}\begin{array}{c}\text { Friday } \\
\text { 04.04.14 }\end{array} \\
13.3\end{array}$} & \multirow{2}{*}{$\begin{array}{c}\text { Saturday } \\
12.04 .14 \\
12.3\end{array}$} \\
\hline & 48.2 & \pm 12.3 & 41.8 & \pm 13.4 & 47.5 & \pm 9.2 & & 7.2 & & & & \\
\hline Hydroxycotinine & 3873.8 & \pm 616.9 & 1919.1 & \pm 204.9 & 2155.9 & \pm 243.6 & 239.5 & 331.7 & 500.1 & 577.7 & 575.3 & 636.3 \\
\hline Atenolol & 165.7 & \pm 22.0 & 134.3 & \pm 21.1 & 136.2 & \pm 6.5 & 45.0 & 75.3 & 53.3 & 53.2 & 95.8 & 77.1 \\
\hline Paracetamol & 6323.7 & \pm 1026.7 & 6335.2 & \pm 153.2 & 4210.4 & \pm 468.2 & 485.6 & 1129.3 & 1703.1 & 1481.7 & 2355.3 & 2209.2 \\
\hline Methylone $^{\mathrm{a}}$ & 11.8 & - & nd & - & nd & - & nd & nd & nd & nd & nd & nd \\
\hline 6-MAM & 25.2 & \pm 10.5 & 6.0 & \pm 10.4 & 2.8 & \pm 2.4 & 8.0 & 16.1 & 25.3 & 23.7 & 32.6 & 39.2 \\
\hline Amphetamine & 51.5 & \pm 10.8 & 38.1 & \pm 7.6 & 51.1 & \pm 4.1 & 76.5 & 124.1 & 91.2 & 53.2 & 91.8 & 56.9 \\
\hline MDMA & 75.5 & \pm 65.5 & 25.7 & \pm 22.3 & 26.8 & \pm 23.8 & nd & nd & nd & nd & nd & nd \\
\hline Methamphetamine & $<\mathrm{LOQ}$ & & 26.3 & \pm 9.7 & 25.8 & \pm 2.4 & 14.2 & 26.3 & 11.6 & 35.2 & 50.1 & 49.6 \\
\hline Benzoylecgonine & 214.3 & \pm 86.8 & 216.3 & \pm 24.1 & 240.9 & \pm 59.1 & 3.5 & 14.5 & 5.8 & 9.0 & 9.0 & 12.2 \\
\hline Ketamine $^{a}$ & nd & - & 5.3 & \pm 9.1 & nd & - & nd & nd & nd & nd & nd & nd \\
\hline Methoxetamine $^{a}$ & 0.8 & \pm 1.3 & nd & - & nd & - & nd & nd & nd & nd & nd & nd \\
\hline Metoprolol & 836.2 & \pm 87.5 & 859.1 & \pm 183.9 & 857.3 & \pm 64.0 & 123.0 & 125.6 & 141.8 & 146.3 & 280.1 & 250.2 \\
\hline Methylphenidate & nd & & nd & & nd & - & 0.6 & 1.2 & 2.7 & 1.3 & 2.7 & 3.0 \\
\hline Cocaine & 92.5 & \pm 35.5 & 77.1 & \pm 18.5 & 88.7 & \pm 23.6 & 0.8 & 2.1 & 3.1 & 2.0 & 3.4 & 4.0 \\
\hline Cocaethylene & 7.3 & \pm 6.7 & 9.8 & \pm 1.3 & 6.1 & \pm 1.7 & nd & nd & nd & nd & nd & nd \\
\hline Propranolol & 3.5 & \pm 0.7 & 14.8 & \pm 1.5 & 0.8 & \pm 0.1 & 4.3 & 13.9 & 20.8 & 13.0 & 22.7 & 26.7 \\
\hline Citalopram & 44.0 & \pm 9.1 & 49.3 & \pm 7.7 & 44.8 & \pm 0.9 & 32.2 & 43.9 & 14.5 & 12.3 & 27.5 & 17.4 \\
\hline Buprenorphine & nd & - & nd & - & nd & - & 214.5 & 292.5 & 96.7 & 81.8 & 183.5 & 115.7 \\
\hline Carbamazepine & 258.3 & \pm 29.2 & 294.6 & \pm 16.6 & 248.0 & \pm 27.1 & 52.1 & 52.2 & 35.0 & 69.2 & 116.7 & 112.7 \\
\hline EDDP & 48.5 & \pm 9.2 & 36.3 & \pm 3.5 & 18.6 & \pm 1.9 & 10.3 & 11.6 & 7.2 & 7.1 & 13.9 & 8.6 \\
\hline Oxazepam & 153.2 & \pm 32.3 & 125.0 & \pm 25.9 & 144.0 & \pm 6.5 & 72.6 & 119.8 & 69.3 & 94.7 & 141.4 & 140.9 \\
\hline Alprazolam & nd & & nd & & nd & & 7.5 & 798.8 & 462.0 & 631.0 & 942.5 & 939.1 \\
\hline Methadone & 13.4 & \pm 2.3 & 16.8 & \pm 4.1 & 14.5 & \pm 2.3 & 1.1 & 2.1 & 3.7 & 1.8 & 3.2 & 3.7 \\
\hline
\end{tabular}




\title{
Target and suspect screening of psychoactive substances in sewage-based samples by UHPLC-QTOF
}

\author{
J.A. Baz-Lombab* ${ }^{\text {ab* Malcolm J. Reid }}{ }^{a}$, Kevin V. Thomas ${ }^{a}$ \\ ${ }^{a}$ Norwegian Institute for Water Research, Gaustadalléen 21, NO-0349 Oslo, Norway \\ bFaculty of Medicine, University of Oslo, PO box 1078 Blindern, 0316 Oslo, Norway \\ * Corresponding author email: jba@niva.no; Tel: +47 98215460
}

Experimental Section

Reagents

Sewage-based samples

Extraction procedure

Figure S-1: Influence of silanisation on the recovery

Figure S-2: Recovery of the studied compounds after the evaporation to dryness

Figure S-3: Effect of the loading volume on the matrix effect

Figure S-4: Detection and identification of N-adamantyl-1-pentylindole-3- carboxamide

Figure S-5: Detection and identification 6-APB/ 5-APB

Figure S-6: Total ion chromatogram for the synthetic cannabinoids

Table S-1: SPE-DEX procedure

Table S- 2: Experimental parameters used for the validation and quantification

Table S-3: Method validation in influent wastewater $(n=6)$

Table S-4: Stability of the spiked analytes in WW

Table S-5: Results obtained for POCIS

Table S-6: Results for target compounds obtained from the pooled urine samples

Table S-7: Occurrence of the suspect candidates in pooled urine samples 


\section{Experimental Section}

\section{Reagents}

The illicit drugs, pharmaceuticals and their metabolites reference substances selected for target analysis were cocaine, cocaethylene, benzoylecgonine, amphetamine, methamphetamine, 3,4-methylenedioxymethamphetamine (MDA), 3,4-methylenedioxyethylamphetamine (MDMA or ecstasy),parahydroxymethamphetamine, para-methoxyamphetamine (PMA), para-methoxy-N-methylamphetamine (PMMA), ketamine, dehydronorketamine, norketamine, methoxetamine, pseudoephedrine, cathinone, methcathinone (ephedrone), methylone, 4'-methyl-N-ethylcathinone (4-MEC), mephedrone (4MMC),methadone, 2-ethylidene-1,5-dimethyl-3,3-diphenylpyrrolidine (EDDP), morphine, 6monoacetylmorphine (6-MAM), fentanyl, buprenorphine, AH-7921, hydroxycotinine, atenolol, salbutamol, gabapentin, paracetamol, metoprolol, methylphenidate, propranolol, citalopram, atomoxetine, carbamazepine, midazolam, nitrazepam, clonazepam, lorazepam, oxazepam, alprazolam, flunitrazepam, etizolam, phenazepam, diazepam, diclofenac, etizolam, carbamazepine, $\Delta$-9-tetrahydrocannabidiol (THC), 11-hydroxy- $\Delta$-9-tetrahydrocannabidiol (OH-THC), 11 -nor-9-carboxy- $\Delta$-9-tetrahydrocannabidiol (THC$\mathrm{COOH}$ ), which were obtained from Nerliens Meszansky (Oslo, Norway) as solutions in methanol $(\mathrm{MeOH})$ or acetonitrile (ACN) at concentrations of $1 \mathrm{mg}$ mL-1. Standard solutions of each compound were made at $100 \mathrm{ng} \mathrm{mL}-1$ in methanol and then diluted into a final mix solution to a concentration of 1 ng $\mathrm{mL}-1$

Synthetic cannabinoids and their selected biomarkers, JWH-073, XLR-11, UR-144, AM-2201, JWH-018, MAM-2201, JWH-122, 5-3-1-Naphthoyl-1H-indol-1-yl-pentanoic acid (JWH-018 N-pentanoic acid), 1-5hydroxypentyl-1H-indol-3-ylnaphthalen-1-yl-methanone (JWH 018 N-5-hydroxypentyl), 4-3-1-naphthoyl1H-indol-1-yl-butanoic acid (JWH-073 N-butanoic acid), 1-4-hydroxybutyl-1H-indol-3-ylnaphthalen-1-ylmethanone (JWH-073 N-4-hydroxybutyl), 1-5-hydroxypentyl-1H-indol-3-yl4-methylnaphthalen-1-ylmethanone (JWH-122 N-5-hydroxypentyl), 1-5-fluoro-4-hydroxypentyl-1H-indol-3-ylnaphthalen-1ylmethanone (AM-2201 N-4-hydroxypentyl), 1-5-hydroxypentyl-1H-indol-3-yl4-methoxyphenylmethanone (RCS-4 N-5-hydroxypentyl) were obtained from Chiron (Trondheim, Norway) in solutions of $1 \mathrm{~mL}$ at $50 \mu \mathrm{g} \mathrm{mL}-1$ in $\mathrm{MeOH}$ or $\mathrm{ACN}$.

Benzo Fury, 1-benzofuran-6-ylpropan-2-amine (6-APB) or 1-benzofuran-5-ylpropane-2-amine (5-APB), and 2NE1/APICA, N-adamantyl-1-pentylindole-3-carboxamide were purchased through online website.

Deuterated standards were purchased from Nerliens Meszansky as solutions of $100 \mathrm{ng} \mathrm{mL}-1$ in $\mathrm{MeOH}$ or ACN and were used as surrogate isotope labelled internal standards (ILIS) for quantification: atenolol-d7, atomoxetine-d7, diclofenac-d4, gabapentin-d10, metoprolol-d7, pregabalin-d6, benzoylecgonine-d3, cocaine-d3, ketamine-d4, norketamine-d4, mephedrone-d3, methcathinone-d3 (ephedrone-d3), methylone-d3, pseudoephedrine-d3, buprenorphine-d4, diazepam-d5, oxazepam-d5, fentanyl-d5, methadone-d3, amphetamine-d5, MDA-d5, MDMA-d5, methamphetamine-d5, THC-OH-d3, THCCOOH-d3. Final standard mix solution was made in methanol at $1 \mathrm{ng} \mathrm{mL}-1$. Finally all the standard solutions were stored in amber glass bottles at $-20^{\circ} \mathrm{C}$.

The ultrapure water was obtained by purifying demineralized water in an Elga Maxima Ultrapure Water purification system (Elga, Lane End, UK). Ammonium formate (for mas spectroscopy, $\geq 99.0 \%$ ), HPLCgrade formic acid (eluent additive for LC-MS) and UHPLC-grade water, $\mathrm{MeOH}$ and ACN were acquired from Sigma-Aldrich, Fluka for HPLC (Oslo, Norway) 


\section{Sewage-based samples}

Influent samples were collected from two wastewater treatment plants (WWTWs); VEAS WWTW in Oslo and Ladehammeren and Høvringen WWTWs in Trondheim Norway.

Nine influent wastewater composite samples were collected during three consecutive weekends from VEAS sewage treatment plant, Oslo Norway, in February 2014. An ISCO Avalanche Portable Refrigerated Sampler (Lincoln, NE, USA) was used to collect the composite samples (50 mL, time proportional) every 30 minutes for the duration of the sampling period. Maximum storage time for the daily composite samples was 3 days at $0.5^{\circ} \mathrm{C}$ in polypropylene bottles. The composite samples were transported every Monday in cooler bags and stored at $-20{ }^{\circ} \mathrm{C}$ until analysis to minimize the degradation of the analytes. The catchment area of VEAS is comprised of $63 \%$ of the Oslo population, around 600,000 inhabitants. The total length of the tunnel is $42.3 \mathrm{~km}$ and the mean residence time in the sewer system is 5 hours (VEAS annual report 13'). The mean influent flow rate for each weekend was 600,820 , 775,327 , and $410,970 \mathrm{~m} 3 /$ day, which is higher that the annual mean $(273,196 \mathrm{~m} 3$ day- 1 in 2013$)$ and was due to increased precipitation during the sampling campaign.

Six influent 24-hour composite wastewater samples were collected during February, March and April of 2014 in two different sewage treatment plant located in Trondheim (Norway). Ladehammeren and Høvringen WWTPs treat the sewage from the city with an estimated population of 180,000 inhabitants. Samples were received frozen and analysed upon reception in the laboratory. Mean influent flow rates are described in the results section.

The robustness of this work was also examined with the analysis of different sewage-based samples. The "Pharmaceuticals" version of the POCIS was made up of Oasis HLB sandwiched between polyethersulphone membranes as described in literature ${ }^{1}$. POCIS were deployed in the sedimentation overflow channel at VEAS, the Oslo WWTP, for two weeks. The study and collection of the pooled urine samples was conducted in Norway during the course of three different music festivals. Portable toilets were available to be used by the festival participants anonymously and no data on the number of users was collected. 


\section{Extraction procedure}

The HLB extraction disks were conditioned by washing and rinsing with methanol $(10 \mathrm{~mL})$ and water $(10$ $\mathrm{mL}$ ). Samples were automatically loaded and filtered (Fast Flow Pre-Filters, Horizon Technology) onto the disks at a flow rate of $100 \mathrm{~mL}$ min-1, and then the disks were washed 4 times with $5 \%$ methanol in water and dried under vacuum for 10 minutes.

Then the samples were loaded directly onto the disk from the sample bottle and prior elution, the disk is air-dried under vacuum providing a shorter operational time. The analytes were finally eluted into a silanised glass vial with 2 cycles of $5 \%$ ammonium hydroxide in methanol and 2 cycles of $5 \%$ acetic acid in methanol. The total program time was approximately 25 minutes. In general this system is more simple, reproducible and cost-effective than the SPE cartridges.

The final extracts were evaporated to around $100 \mu \mathrm{L}$ under a gentle nitrogen stream at $35^{\circ} \mathrm{C}$ and reconstructed in $400 \mu \mathrm{L}$ of $25 \%$ methanol aqueous solution. An aliquot was centrifuged and then $5 \mu \mathrm{L}$ were injected into the UHPLC-QTOF. The general procedure is also described in the Table S-1.

The "Pharmaceuticals" version of the POCIS were deployed in the wastewater treatment plant and subsequently the sorbent was removed and introduced in an empty SPE cartridge with Mili-Q water, washed twice with $6 \mathrm{~mL}$ of $5 \%$ methanol in water and finally eluted with $6 \mathrm{~mL}$ of $0.5 \%$ ammonium hydroxide in methanol and $6 \mathrm{~mL}$ of $0.5 \%$ acetic in methanol. The eluent was evaporated under a stream of nitrogen and diluted to $1.5 \mathrm{~mL}$ with $13 \%$ methanol in water. $100 \mathrm{ng}$ of the ILIS mix was added during the washing step.

The pooled urine samples were firstly homogenized and then centrifuged during 20 minutes at $2500 \mathrm{rpm}$. $5 \mathrm{~mL}$ of the supernatant was collected, spiked with $100 \mathrm{ng}$ of the ILIS mix and introduced into the HLB cartridge. Washed with $2 \times 6 \mathrm{~mL} 5 \%$ methanol in water, the elution was finally done with $6 \mathrm{~mL}$ of $0.5 \%$ ammonium hydroxide in methanol and $6 \mathrm{~mL}$ of $0.5 \%$ acetic in methanol. The eluent was evaporated under a stream of nitrogen and diluted to $1 \mathrm{~mL}$ with $13 \%$ methanol in water. 


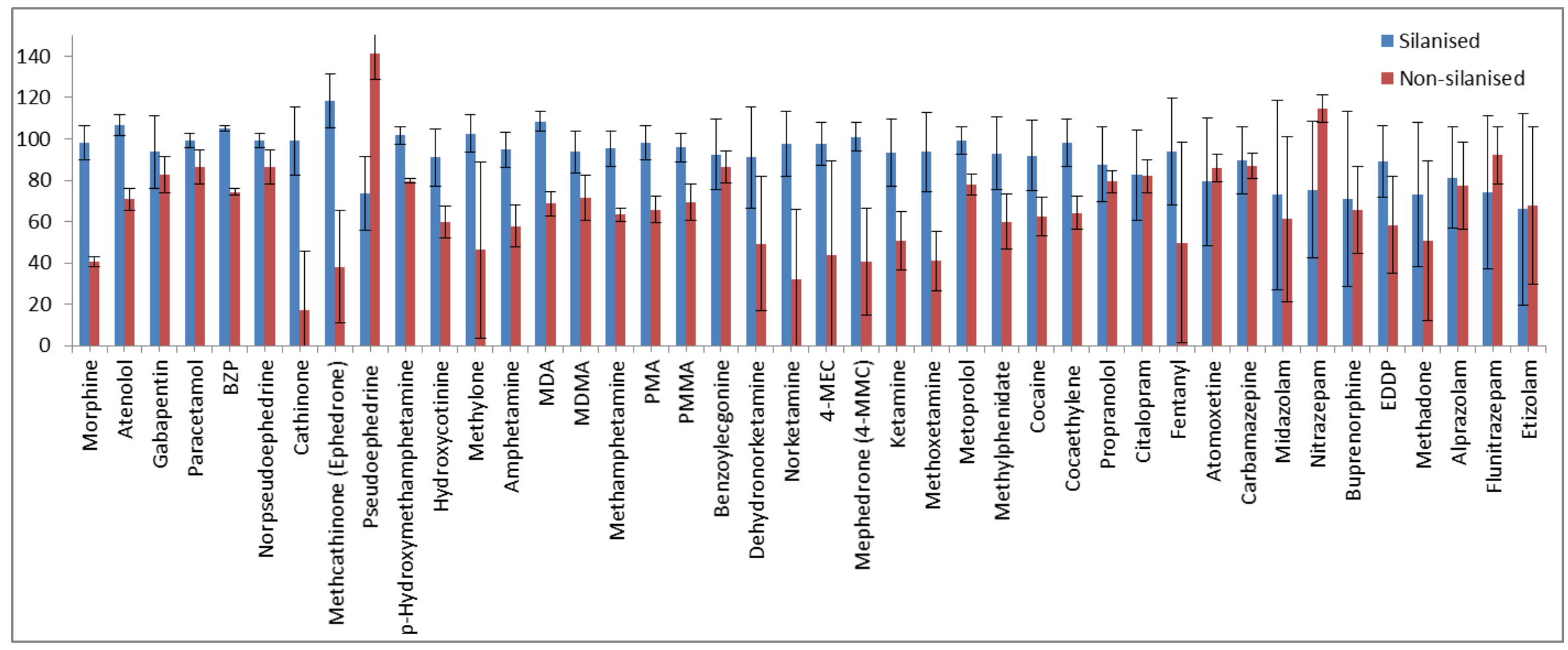

Figure S-1

Influence of silanisation on the recovery (\%) of the studied compounds during the evaporation in the SPE extract vials. Recovery of $100 \mathrm{~mL}$ of MiliQ water spiked with $100 \mathrm{ng}$ of the standard mix solution using HLB cartridges $(n=3)$ 


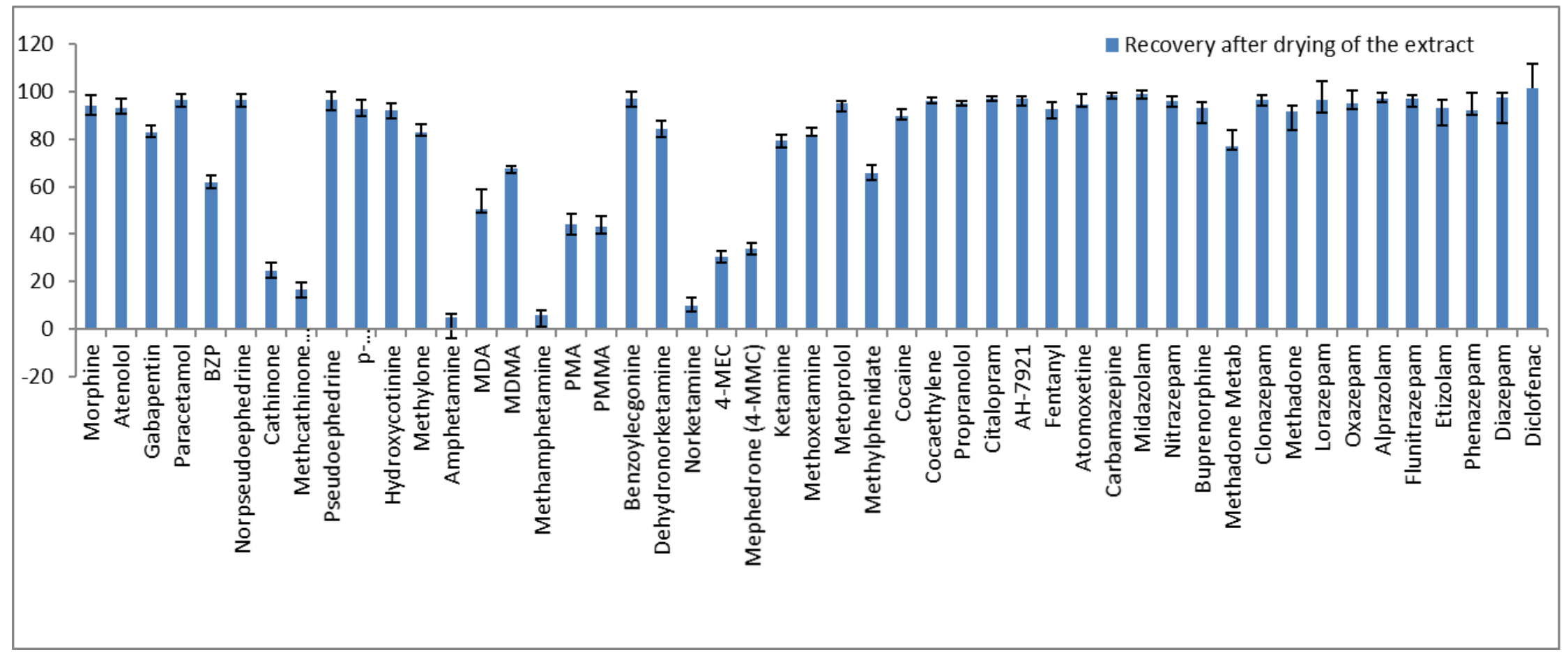

Figure S-2

Recovery of the studied compounds after the evaporation to dryness at $50^{\circ} \mathrm{C}$ of a $5 \mathrm{~mL}$ methanol solution with $100 \mathrm{ng}$ of the mix standard solution ( $\mathrm{n}=3$ ) 


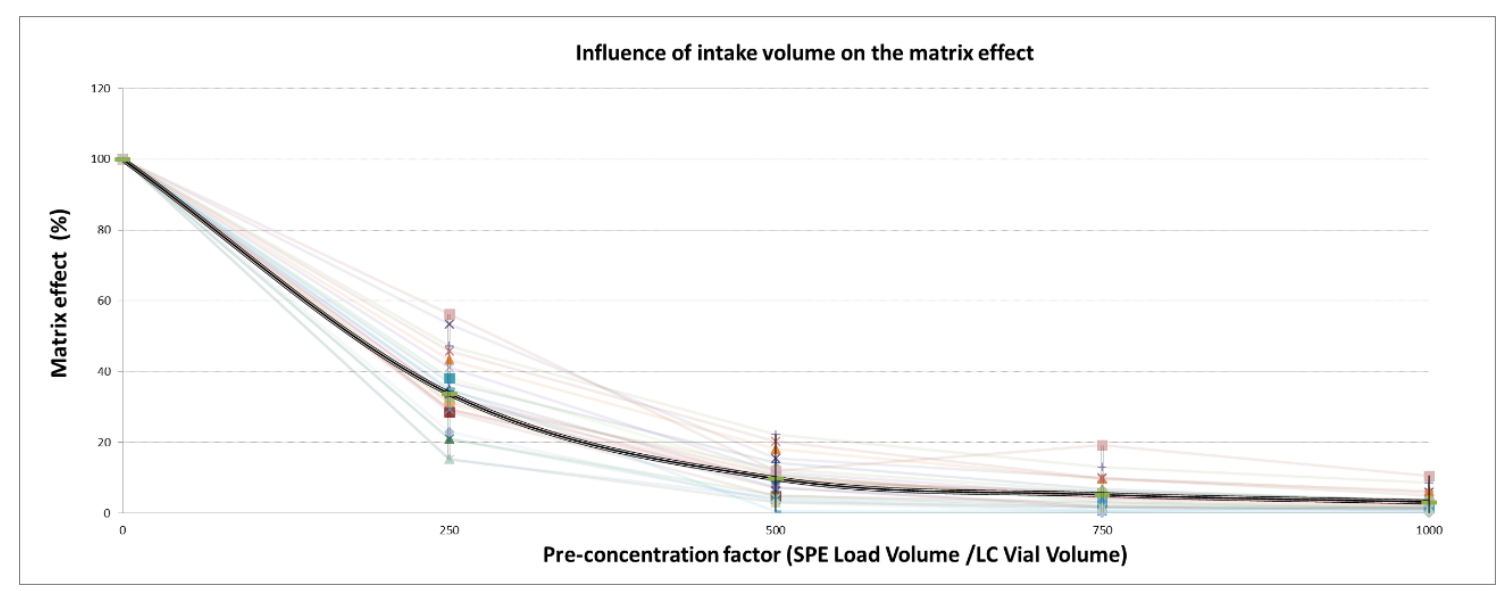

Figure S-3

Effect of the loading volume on the matrix effect. Matrix suppression average for 25 ILIS spiked into the extracts of 3 different wastewater samples. The loading volumes were $100,200,300$, and $400 \mathrm{~mL}$ and the final extract was reconstituted volume of $400 \mu \mathrm{L}$ 


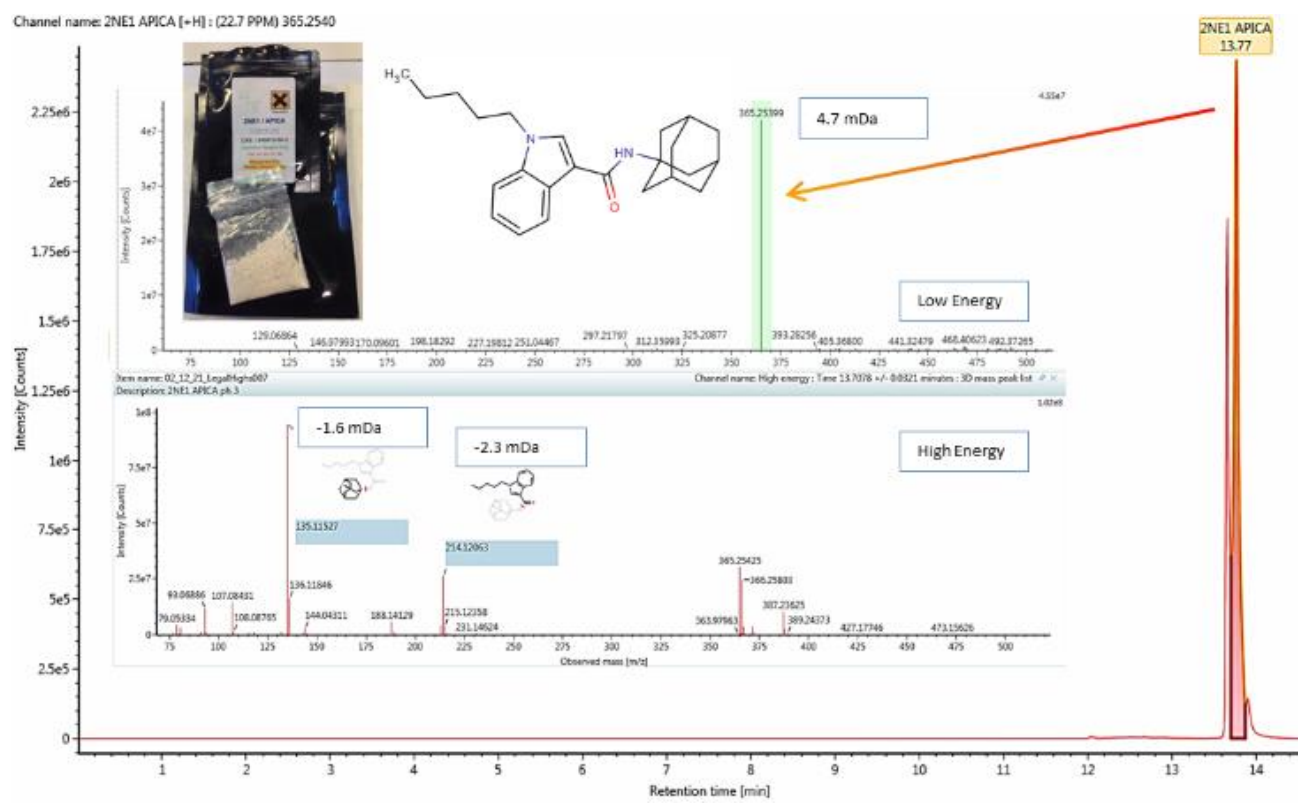

Figure S-1

Detection and identification of $\mathrm{N}$-adamantyl-1-pentylindole-3-carboxamide in a sample purchased on internet under the name of "2NE1/APICA". Extracted ion chromatogram of the 2NE1/APICA powder sample and the spectra in low and high energy time-of-flight. Possible fragment structures assigned manually by UNIFI 


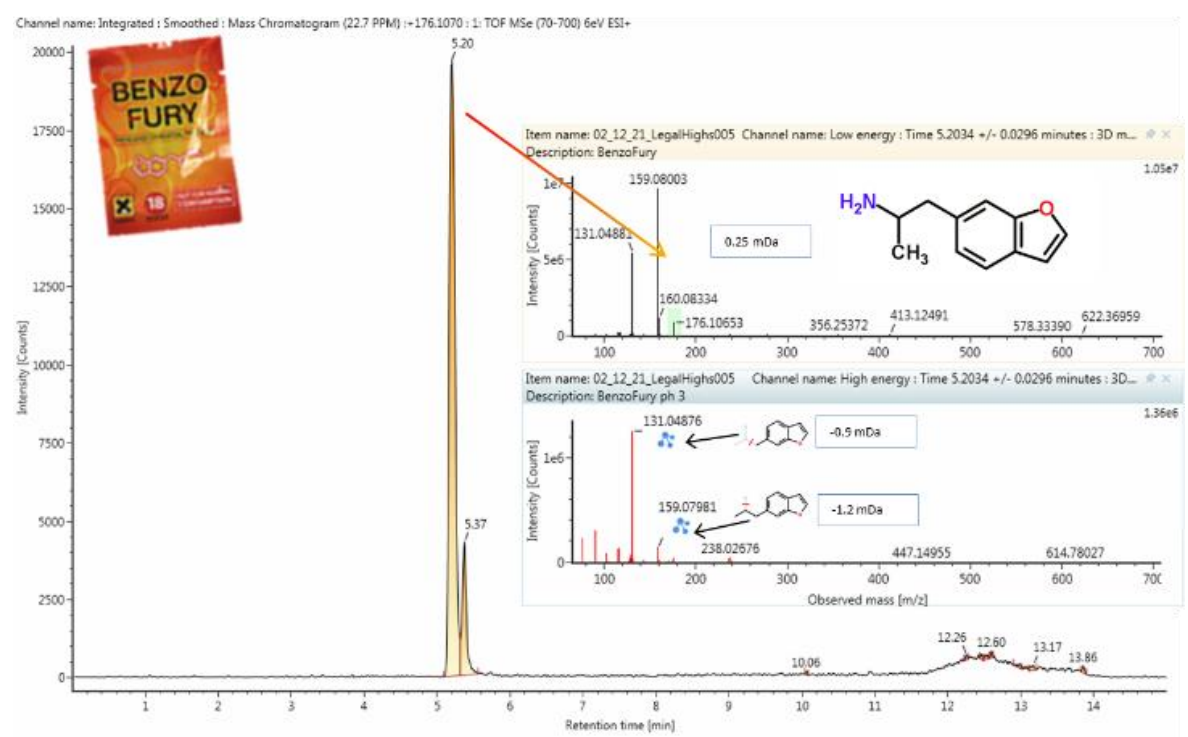

Figure S-2

Detection and identification of 6-(2-aminopropyl)benzofuran (6-APB)/ 5-APB (5-(2-aminopropyl)benzofuran (5-APB) in a sample purchased on internet under the name of "Benzo Fury". Extracted ion chromatogram of the Benzo Fury tablet sample and the spectra in low and high energy time-of-flight. Possible fragment structures assigned manually by UNIFI 

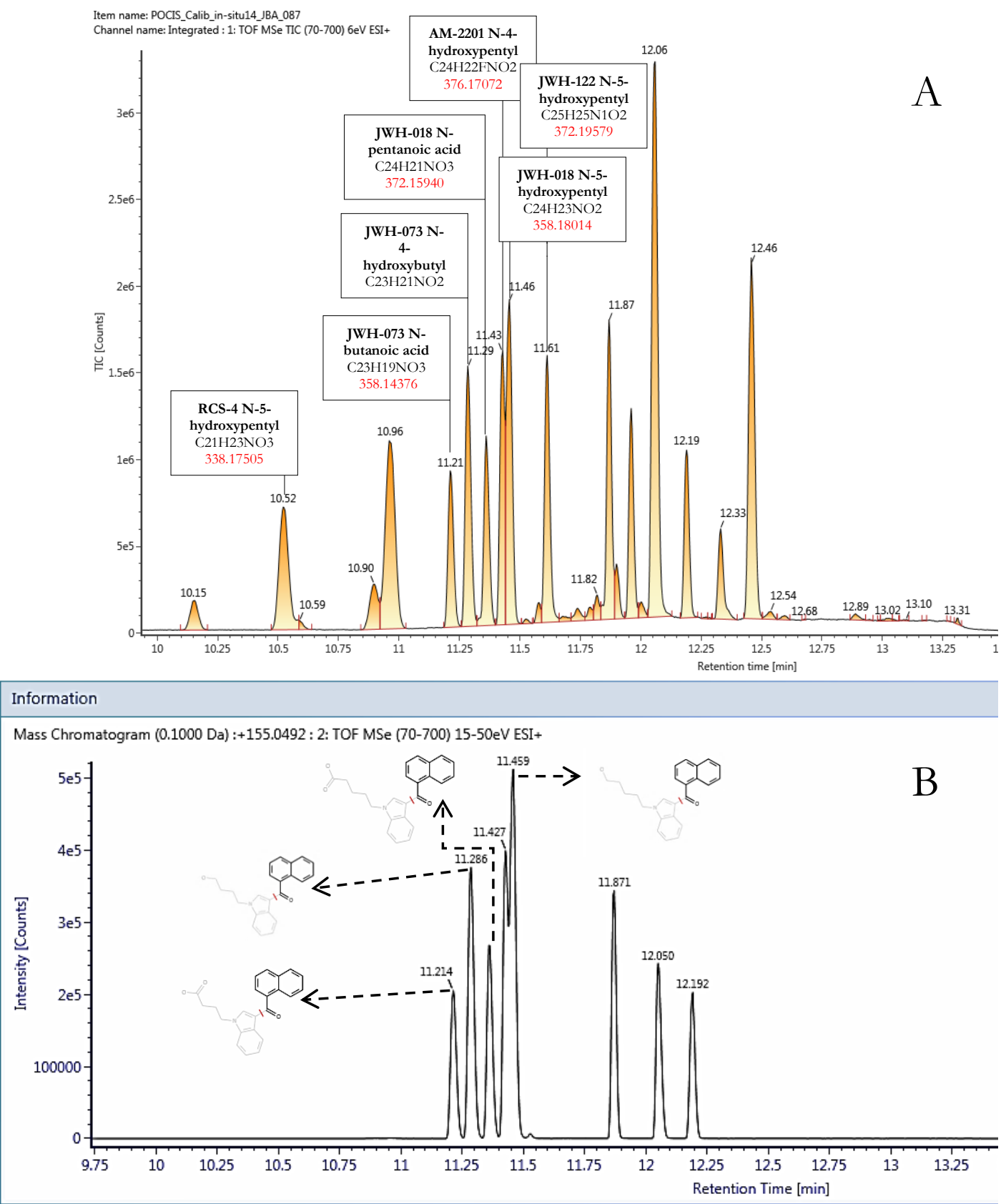

Figure S-6

Total ion chromatogram for the 14 synthetic cannabinoids of a standard mix at $250 \mathrm{ng} / \mathrm{mL}$ with the description of the main metabolites A). Common fragment (155.0492) chromatogram from four synthetic cannabinoids and their four metabolites B) 
Table S-1

SPE-DEX procedure

\begin{tabular}{lcll}
\hline Step & Solvent & Soak time & Dry time \\
\hline Preconditioning & Methanol (2 times) & $20 \mathrm{~s}$ & $10 \mathrm{~s}$ \\
& Water & $20 \mathrm{~s}$ & $10 \mathrm{~s}$ \\
& Water & $20 \mathrm{~s}$ & $0 \mathrm{~s}$ \\
\hline Sample loading & - & - & - \\
\hline Washing & $5 \%$ methanol/water(4 times) & $10 \mathrm{~s}$ & $30 \mathrm{~s}$ \\
Disk drying & - & - & $10 \mathrm{~min}$ \\
\hline Eluting & $5 \%$ ammonium hydroxide in methanol & $30 \mathrm{~s}$ & $30 \mathrm{~s}$ \\
& $5 \%$ ammonium hydroxide in methanol & $30 \mathrm{~s}$ & $2 \mathrm{~min}$ \\
& $5 \%$ acetic acid in methanol & $30 \mathrm{~s}$ & $30 \mathrm{~s}$ \\
& $5 \%$ acetic acid in methanol & $30 \mathrm{~s}$ & $2 \mathrm{~min}$ \\
\hline
\end{tabular}


Table S- 2

Experimental parameters used for the validation and quantification of the target compounds: chromatographic retention times, exact mass of the protonated target compounds, validated linear range, correlation coefficient, mass error and the results of the instrumental performance

Accuracy $(\%)$

Precision (RSD\%)

\begin{tabular}{|c|c|c|c|c|c|c|c|c|c|c|c|c|c|}
\hline \multirow[t]{2}{*}{ Compound } & \multirow[t]{2}{*}{$t_{R}$} & \multirow{2}{*}{$\begin{array}{l}\text { Precursor ion } \\
\qquad[\mathrm{M}+\mathrm{H}]^{+}\end{array}$} & \multirow{2}{*}{$\begin{array}{l}\text { Linear range } \\
\qquad\left(n g m L^{-1}\right)\end{array}$} & \multirow[t]{2}{*}{ Linearity $R^{2}$} & \multirow{2}{*}{$\begin{array}{l}\text { Mass Errot } \\
\text { (mDa/ppm) }\end{array}$} & \multicolumn{2}{|c|}{ Intra-day } & \multicolumn{2}{|c|}{ Inter-day } & \multicolumn{2}{|c|}{ Intra-day } & \multicolumn{2}{|c|}{ Inter-day } \\
\hline & & & & & & Low & High & Low & High & Low & High & Low & High \\
\hline Morphine & 1.17 & 286.14376 & $0,5-100$ & 0.995 & $-0.08 /-0.29$ & 94 & 101 & 94 & 101 & 1 & 1 & 3 & 2 \\
\hline Hydroxycotinine & 1.17 & 193.09714 & $0,5-400$ & 0.999 & $0.09 / 046$ & 92 & 97 & 88 & 98 & 3 & 2 & 5 & 5 \\
\hline Atenolol & 1.26 & 267.1703 & $0,5-200$ & 1.000 & $-0.05 /-0.2$ & 108 & 99 & 108 & 99 & 3 & 0 & 3 & 0 \\
\hline Salbutamol & 1.28 & 240.1594 & $1-100$ & 0.995 & $-0.17 /-0.67$ & 108 & 100 & 107 & 99 & 2 & 1 & 5 & 2 \\
\hline p-Hydroxymethamphetamine & 1.31 & 166.12263 & $2-100$ & 0.998 & $-0.17 /-0.99$ & 96 & 102 & 99 & 101 & 7 & 2 & 7 & 2 \\
\hline Paracetamol & 1.62 & 152.07059 & $2-400$ & 0.998 & $0 /-0.02$ & 98 & 101 & 104 & 101 & 3 & 2 & 9 & 5 \\
\hline Cathinone & 1.75 & 150.09133 & $5-400$ & 0.991 & $-0.08 /-0.52$ & 93 & 104 & 92 & 104 & 3 & 2 & 3 & 1 \\
\hline Gabapentin & 1.8 & 172.13319 & $5-200$ & 0.995 & $-0.04 /-0.26$ & 110 & 102 & 108 & 99 & 3 & 1 & 2 & 6 \\
\hline Pseudoephedrine & 1.96 & 166.12263 & $5-400$ & 0.998 & $-0.31 /-2.07$ & 96 & 99 & 94 & 99 & 9 & 1 & 8 & 4 \\
\hline Methcathinone (Ephedrone) & 1.98 & 164.10698 & $1-400$ & 0.998 & $-0.35 /-2.49$ & 93 & 100 & 96 & 100 & 1 & 0 & 3 & 0 \\
\hline Methylone & 2.16 & 208.09681 & $0,25-400$ & 0.999 & $0.03 / 0.12$ & 110 & 100 & 109 & 100 & 4 & 0 & 3 & 0 \\
\hline 6-MAM & 2.29 & 328.15432 & $0,5-400$ & 0.998 & $-0.01 /-0.05$ & 95 & 102 & 98 & 101 & 3 & 1 & 4 & 0 \\
\hline Amphetamine & 2.41 & 136.11207 & $5-400$ & 0.993 & $-0.15 /-1.31$ & 88 & 104 & 98 & 104 & 5 & 1 & 12 & 1 \\
\hline MDA & 2.47 & 180.10189 & $10-200$ & 0.994 & $0.04 / 0.29$ & 109 & 104 & 108 & 103 & 6 & 4 & 6 & 4 \\
\hline PMA & 2.53 & 166.12263 & $2-400$ & 0.999 & $-0.42 /-2.59$ & 110 & 100 & 107 & 100 & 2 & 1 & 5 & 1 \\
\hline Methamphetamine & 2.72 & 150.12771 & $2-200$ & 0.999 & $-0.16 /-1.10$ & 105 & 101 & 104 & 100 & 4 & 1 & 5 & 1 \\
\hline
\end{tabular}




\begin{tabular}{|c|c|c|c|c|c|c|c|c|c|c|c|}
\hline MDMA & 2.75 & 194.11754 & $2-400$ & 0.999 & $-0.03 /-0.16$ & 100 & 100 & 100 & 100 & 4 & \\
\hline Dehydronorketamine & 2.89 & 222.06801 & $1-400$ & 0.998 & $0.22 / 0.98$ & 106 & 99 & 108 & 97 & 7 & \\
\hline PMMA & 2.98 & 180.13828 & $2-400$ & 1.000 & $-0.42 /-2.59$ & 101 & 100 & 100 & 100 & 3 & \\
\hline Benzoylecgonine & 3.07 & 290.13867 & $0,25-400$ & 0.999 & $0.01 / 0.07$ & 94 & 101 & 95 & 101 & 2 & \\
\hline Mephedrone (4-MMC) & 3.19 & 178.12263 & $2-400$ & 0.999 & $-0.14 /-0.86$ & 107 & 99 & 105 & 99 & 8 & \\
\hline Norketamine & 3.21 & 224.08366 & $5-200$ & 0.999 & $0.12 / 0.58$ & 109 & 102 & 104 & 101 & 4 & \\
\hline Ketamine & 3.45 & 238.09931 & $0,5-400$ & 1.000 & $-0.35 /-2.49$ & 98 & 99 & 97 & 99 & 3 & \\
\hline 4-MEC & 3.7 & 192.13828 & $0,5-400$ & 1.000 & $-0.09 /-0.48$ & 97 & 99 & 98 & 99 & 2 & \\
\hline Methoxetamine & 4.12 & 248.16449 & $0,25-400$ & 1.000 & $-0.06 /-0.21$ & 105 & 99 & 105 & 99 & 7 & \\
\hline Metoprolol & 4.19 & 268.1907 & $0,25-400$ & 0.999 & $-0.16 /-0.61$ & 102 & 100 & 101 & 100 & 1 & \\
\hline Methylphenidate & 4.32 & 234.14884 & $0,25-400$ & 1.000 & $0 /-0.05$ & 98 & 100 & 94 & 100 & 3 & \\
\hline Cocaine & 4.83 & 304.15432 & $0,25-200$ & 1.000 & $0 / 0.03$ & 97 & 101 & 97 & 101 & 2 & \\
\hline Cocaethylene & 5.87 & 318.16997 & $0,25-400$ & 1.000 & $0.04 /-0.13$ & 96 & 102 & 96 & 101 & 1 & \\
\hline Propranolol & 6.28 & 260.16449 & $0,25-200$ & 0.998 & $-0.04 /-0.13$ & 105 & 99 & 105 & 94 & 2 & \\
\hline Fentanyl & 6.63 & 337.22742 & $0,25-400$ & 1.000 & $-0.24 /-0.69$ & 107 & 99 & 105 & 99 & 4 & \\
\hline АН-7921 & 7.02 & 329.11818 & $0,25-400$ & 1.000 & $-0.1 /-0.33$ & 110 & 99 & 113 & 100 & 4 & \\
\hline Citalopram & 7.07 & 325.17105 & $0,25-200$ & 0.997 & $-0.14 /-0.42$ & 100 & 100 & 100 & 100 & 4 & \\
\hline Midazolam & 7.14 & 326.08547 & $0,25-400$ & 0.999 & $-0.03 /-0.1$ & 106 & 99 & 108 & 97 & 7 & \\
\hline Buprenorphine & 7.38 & 468.31081 & $0,25-400$ & 1.000 & $0.11 / 0.25$ & 101 & 100 & 100 & 100 & 3 & \\
\hline Carbamazepine & 7.54 & 237.10223 & $0,25-200$ & 1.000 & $0.19 / 0.75$ & 94 & 101 & 95 & 101 & 2 & \\
\hline EDDP & 7.75 & 278.19031 & $0,25-400$ & 0.999 & $-0.1 /-0.33$ & 107 & 99 & 105 & 99 & 8 & \\
\hline Atomoxetine & 7.81 & 256.16958 & $1-200$ & 0.999 & $-0.02 /-0.07$ & 109 & 102 & 104 & 101 & 4 & \\
\hline
\end{tabular}




\begin{tabular}{|c|c|c|c|c|c|c|c|c|c|c|c|c|c|}
\hline Nitrazepam & 8.16 & 282.08731 & $1-400$ & 0.998 & $-0.09 /-0.33$ & 98 & 99 & 97 & 99 & 3 & 1 & 4 & 1 \\
\hline Oxazepam & 8.22 & 287.05817 & $2-400$ & 0.997 & $-0.51 /-1.78$ & 97 & 99 & 98 & 99 & 2 & 1 & 2 & 1 \\
\hline Clonazepam & 8.5 & 316.04833 & $1-400$ & 0.998 & $-0.35 /-1.09$ & 105 & 99 & 105 & 99 & 7 & 0 & 5 & 0 \\
\hline Lorazepam & 8.6 & 321.0192 & $5-400$ & 0.994 & $-0.22 /-0.69$ & 102 & 100 & 101 & 100 & 1 & 1 & 2 & 1 \\
\hline Alprazolam & 8.83 & 309.09014 & $0,25-200$ & 0.996 & $-0.04 /-0.15$ & 98 & 100 & 94 & 100 & 3 & 1 & 5 & 1 \\
\hline Methadone & 8.89 & 310.21652 & $0,25-400$ & 0.999 & $0.39 / 1.24$ & 97 & 101 & 97 & 101 & 2 & 0 & 1 & 0 \\
\hline Flunitrazepam & 9.19 & 314.09353 & $1-400$ & 1.000 & $-0.07 /-0.18$ & 96 & 102 & 96 & 101 & 1 & 1 & 1 & 1 \\
\hline Etizolam & 9.53 & 343.07786 & $0,25-200$ & 1.000 & $-0.08 /-0.26$ & 105 & 99 & 105 & 94 & 2 & 1 & 2 & 6 \\
\hline Phenazepam & 10.1 & 348.97382 & $2-400$ & 0.998 & $-0.28 /-0.82$ & 107 & 99 & 105 & 99 & 4 & 0 & 5 & 1 \\
\hline Diazepam & 10.91 & 285.07891 & $0,25-200$ & 1.000 & $0.05 / 0.19$ & 110 & 99 & 113 & 100 & 4 & 0 & 5 & 2 \\
\hline Diclofenac & 11.4 & 296.02395 & $5-400$ & 0.997 & $-0.02 /-0.08$ & 100 & 100 & 100 & 100 & 4 & 1 & 3 & 1 \\
\hline THC-OH & 11.83 & 331.22675 & $5-400$ & 0.995 & $-0.17 /-0.56$ & 106 & 99 & 108 & 97 & 7 & 0 & 7 & 2 \\
\hline THC-COOH & 11.85 & 345.20602 & $5-400$ & 0.992 & $0.17 / 0.5$ & 101 & 100 & 100 & 100 & 3 & 0 & 3 & 1 \\
\hline
\end{tabular}


Table S-3

Method validation in influent wastewater $(n=6)$

\begin{tabular}{|c|c|c|c|c|c|c|c|c|c|c|}
\hline \multirow[b]{2}{*}{ Compound } & \multirow[b]{2}{*}{ Internal Standard } & \multirow[b]{2}{*}{$\begin{array}{c}\text { Matrix } \\
\text { Suppression }\end{array}$} & \multicolumn{4}{|c|}{ Recovery } & \multirow[b]{2}{*}{$\begin{array}{c}\text { Instrumental LOQ } \\
\quad(\text { ng } m L-1)\end{array}$} & \multirow[b]{2}{*}{$\begin{array}{l}\text { Theoretical LOQ } \\
\quad\left(n g L^{-1}\right)\end{array}$} & & \multirow[b]{2}{*}{ Information } \\
\hline & & & $100 \mathrm{ng} \mathrm{L}^{-1}$ & $\mathrm{CV}(\%)$ & $\begin{array}{c}600 \\
\operatorname{ng~L}_{1}^{-}\end{array}$ & $\mathrm{CV}(\%)$ & & & & \\
\hline Morphine & Atenolol-d 7 & 38 & 73 & 9 & 68 & 10 & 0.40 & 4.2 & & \\
\hline Hydroxycotinine & Atenolol-d7 & 38 & 120 & 19 & 88 & 17 & 0.57 & 5.9 & * & high concentrations in "blank" \\
\hline Atenolol & Atenolol-d 7 & 38 & 92 & 2 & 91 & 12 & 0.35 & 3.7 & & \\
\hline Salbutamol & Atenolol-d 7 & 38 & 88 & 7 & 90 & 8 & 0.81 & 8.5 & & \\
\hline pHydromethome & Atenolol-d 7 & 38 & - & - & - & - & 1.34 & 14.0 & * & high matrix suppression \\
\hline Paracetamol & Atenolol-d 7 & 38 & - & - & - & - & 1.28 & 13.4 & $*$ & high concentration in "blank" \\
\hline Cathinone & Methcathinone- $\mathrm{d}_{3}$ & 34 & $\sim \mathrm{LOQ}$ & $\sim \mathrm{LOQ}$ & 51 & 2 & 5.69 & 67.5 & & \\
\hline Gatin & Gabapentin- $\mathrm{d}_{10}$ & 30 & - & - & - & - & 3.79 & 51.2 & * & high matrix suppression \\
\hline Pseudoephedrine & Methcathinone- $\mathrm{d}_{3}$ & 34 & 101 & 5 & 118 & 5 & 3.10 & 36.7 & & \\
\hline Methcathinone (Ephedrone) & Methcathinone- $\mathrm{d}_{3}$ & 34 & 85 & 7 & 94 & 1 & 0.85 & 10.0 & & \\
\hline Methylone & Methylone- $\mathrm{d}_{3}$ & 37 & 77 & 2 & 98 & 3 & 0.04 & 0.4 & & \\
\hline 6-MAM & Methylone- $\mathrm{d}_{3}$ & 37 & 72 & 5 & 81 & 4 & 0.30 & 3.2 & & \\
\hline Amphetamine & Amphetamine- $\mathrm{d}_{5}$ & 14 & 77 & 9 & 90 & 13 & 3.06 & 85.6 & & \\
\hline MAA & $\mathrm{MDA}-\mathrm{d}_{5}$ & 26 & - & - & - & - & 11.95 & 187.0 & * & high matrix suppression \\
\hline PMA & MDMA-d 5 & 26 & 57 & 5 & 77 & 8 & 1.03 & 16.1 & & \\
\hline MDMA & MDMA-d ${ }_{5}$ & 26 & 69 & 4 & 69 & 3 & 1.53 & 24.0 & & \\
\hline Methamphetamine & Methamphetamine- $\mathrm{d}_{5}$ & 11 & 77 & 10 & 83 & 5 & 1.51 & 54.1 & & \\
\hline
\end{tabular}




\begin{tabular}{|c|c|c|c|c|c|c|c|c|}
\hline Dehydronorketamine & Norketamine- $\mathrm{d}_{4}$ & 33 & 48 & 2 & 54 & 4 & 0.65 & 8.0 \\
\hline PMMA & MDMA-d ${ }_{5}$ & 26 & 74 & 8 & 79 & 2 & 0.90 & 14.0 \\
\hline Benzoylecgonine & Benzoylecgonine- $\mathrm{d}_{3}$ & 51 & 102 & 9 & 107 & 7 & 0.12 & 0.9 \\
\hline Mephedrone (4-MMC) & Mephedrone- $\mathrm{d} 3$ & 18 & 61 & 2 & 74 & 6 & 1.16 & 26.3 \\
\hline Norketamine & Norketamine- $\mathrm{d}_{4}$ & 33 & 76 & 5 & 76 & 11 & 2.89 & 35.5 \\
\hline Ketamine & Ketamine- $\mathrm{d}_{4}$ & 41 & 80 & 4 & 100 & 6 & 0.26 & 2.5 \\
\hline 4-MEC & Ketamine- $\mathrm{d}_{4}$ & 41 & 73 & 4 & 91 & 5 & 0.28 & 2.7 \\
\hline Methoxetamine & Metoprolol-d $\mathrm{d}_{7}$ & 40 & 32 & 7 & 41 & 3 & 0.19 & 1.9 \\
\hline Metoprolol & Metoprolol-d $\mathrm{d}_{7}$ & 40 & 84 & 12 & 95 & 6 & 0.11 & 1.1 \\
\hline Methylphenidate & Metoprolol-d 7 & 40 & 47 & 9 & 51 & 9 & 0.15 & 1.5 \\
\hline Cocaine & Cocaine- $\mathrm{d}_{3}$ & 52 & 93 & 11 & 103 & 7 & 0.07 & 0.6 \\
\hline Cocaethylene & Cocaine- $\mathrm{d}_{3}$ & 52 & 90 & 9 & 101 & 6 & 0.11 & 0.9 \\
\hline Propranolol & Fentanyl-d 5 & 33 & 102 & 18 & 119 & 11 & 0.06 & 0.8 \\
\hline AH-7921 & Fentanyl-d ${ }_{5}$ & 33 & 97 & 5 & 108 & 12 & 0.20 & 2.4 \\
\hline Citalopram & Buprenorphine- $\mathrm{d}_{4}$ & 24 & 72 & 14 & 84 & 10 & 0.09 & 1.5 \\
\hline Fentanyl & Fentanyl-d 5 & 33 & 90 & 14 & 112 & 3 & 0.14 & 1.7 \\
\hline Midazolam & Buprenorphine- $\mathrm{d}_{4}$ & 24 & 98 & 3 & 119 & 6 & 0.09 & 1.5 \\
\hline Buprenorphine & Buprenorphine- $\mathrm{d}_{4}$ & 24 & 86 & 7 & 115 & 4 & 0.15 & 2.5 \\
\hline Carbamazepine & Buprenorphine- $\mathrm{d}_{4}$ & 24 & 118 & 6 & 130 & 6 & 0.14 & 2.3 \\
\hline EDDP & Atomoxetine-d $\mathrm{d}_{7}$ & 23 & 77 & 16 & 103 & 11 & 0.11 & 2.0 \\
\hline Atomoxetine & Atomoxetine- $\mathrm{d}_{7}$ & 23 & 71 & 1 & 62 & 10 & 0.62 & 10.8 \\
\hline Nitrazepam & Oxazepam-d5 & 78 & 76 & 3 & 93 & 5 & 0.74 & 3.8 \\
\hline
\end{tabular}




\begin{tabular}{|c|c|c|c|c|c|c|c|c|c|c|}
\hline Clonazepam & Oxazepam-d5 & 78 & 57 & 1 & 46 & 1 & 1.14 & 5.9 & & \\
\hline Oxazepam & Oxazepam-d5 & 78 & 113 & 15 & 104 & 14 & 2.23 & 11.5 & & \\
\hline Lorazepam & Oxazepam-d5 & 78 & 86 & 3 & 87 & 2 & 2.31 & 11.9 & & \\
\hline Alprazolam & Oxazepam- $\mathrm{d}_{5}$ & 78 & 75 & 15 & 100 & 14 & 0.16 & 0.8 & & \\
\hline Methadone & Methadone- $\mathrm{d}_{3}$ & 41 & 97 & 8 & 96 & 5 & 0.04 & 0.4 & & \\
\hline Flunitrazepam & Diazepam- $\mathrm{d}_{5}$ & 19 & 73 & 7 & 100 & 15 & 0.71 & 15.2 & & \\
\hline Etizolam & Diazepam- $\mathrm{d}_{5}$ & 19 & 66 & 13 & 84 & 10 & 0.09 & 2.0 & & \\
\hline Phenazepam & Diazepam- $\mathrm{d}_{5}$ & 19 & 104 & 9 & 118 & 9 & 1.50 & 32.2 & & \\
\hline Diazepam & Diazepam- $\mathrm{d}_{5}$ & 19 & 125 & 7 & 110 & 14 & 0.10 & 2.2 & & \\
\hline Diclofenae & Diazepam- $\mathrm{d}_{5}$ & 19 & - & - & - & - & 2.94 & 62.9 & * & high matrix suppression \\
\hline ТНС ОН & THC-OH-d 3 & 19 & - & - & - & - & 2.41 & 50.3 & * & high matrix suppression \\
\hline THC-СOOH & THC-COOH-d 3 & 17 & - & - & - & - & 3.22 & 76.7 & * & high matrix suppression \\
\hline
\end{tabular}


Table S-4

Stability of the spiked analytes $\left(1000 \mathrm{ng} \mathrm{L}^{-1}\right)$ in influent WW $(\mathrm{n}=3)$ at $\mathrm{pH}$ 7. Difference $(\%) / \pm \mathrm{SD}$

\begin{tabular}{|c|c|c|c|c|c|c|}
\hline \multirow{3}{*}{$\begin{array}{l}\text { Compound } \\
\text { Morphine }\end{array}$} & \multicolumn{6}{|c|}{ Peak Area Difference $(\%) \pm$ SD } \\
\hline & \multicolumn{2}{|c|}{$5 \mathrm{~h}$} & \multicolumn{2}{|c|}{$8 \mathrm{~h}$} & \multicolumn{2}{|c|}{$120 \mathrm{~h}$} \\
\hline & -24 & \pm 20 & -5 & \pm 17 & 83 & \pm 12 \\
\hline Hydroxycotinine & 22 & \pm 5 & 82 & \pm 1 & 54 & \pm 4 \\
\hline Atenolol & -9 & \pm 5 & -10 & \pm 4 & -20 & \pm 2 \\
\hline Salbutamol & 9 & \pm 10 & 1 & \pm 9 & -3 & \pm 8 \\
\hline p-Hydroxymethamphetamine & 7 & \pm 14 & -1 & \pm 9 & 70 & \pm 22 \\
\hline Paracetamol & -1 & \pm 14 & -8 & \pm 8 & -88 & \pm 8 \\
\hline Gabapentin & -9 & \pm 25 & -28 & \pm 8 & -32 & \pm 11 \\
\hline Pseudoephedrine & 9 & \pm 10 & 13 & \pm 15 & 0 & \pm 11 \\
\hline Methcathinone (Ephedrone) & -4 & \pm 18 & -8 & \pm 22 & -33 & \pm 11 \\
\hline Methylone & 6 & \pm 62 & -27 & \pm 21 & -53 & \pm 12 \\
\hline 6-MAM & -14 & \pm 7 & -16 & \pm 8 & -21 & \pm 7 \\
\hline Amphetamine & 14 & \pm 3 & 23 & \pm 6 & 33 & \pm 7 \\
\hline MDA & 15 & \pm 11 & -2 & \pm 18 & -96 & \pm 18 \\
\hline PMA & 3 & \pm 20 & 16 & \pm 7 & 10 & \pm 7 \\
\hline MDMA & 29 & \pm 36 & 6 & \pm 6 & 12 & \pm 6 \\
\hline Methamphetamine & -10 & \pm 8 & -15 & \pm 33 & 23 & \pm 29 \\
\hline Dehydronorketamine & -11 & \pm 11 & -7 & \pm 20 & -13 & \pm 18 \\
\hline PMMA & -4 & \pm 33 & 17 & \pm 9 & 6 & \pm 10 \\
\hline Benzoylecgonine & 19 & \pm 8 & 30 & \pm 7 & 56 & \pm 8 \\
\hline Mephedrone (4-MMC) & -1 & \pm 1 & 3 & \pm 1 & 7 & \pm 1 \\
\hline Norketamine & -12 & \pm 6 & -24 & \pm 17 & -25 & \pm 20 \\
\hline Ketamine & 4 & \pm 27 & -13 & \pm 8 & -25 & \pm 5 \\
\hline 4-MEC & 5 & \pm 16 & -2 & \pm 18 & -32 & \pm 12 \\
\hline Methoxetamine & -4 & \pm 4 & -6 & \pm 10 & 13 & \pm 0 \\
\hline Metoprolol & -6 & \pm 3 & -9 & \pm 3 & -5 & \pm 3 \\
\hline Methylphenidate & -10 & \pm 11 & -32 & \pm 4 & -88 & \pm 11 \\
\hline Cocaine & -9 & \pm 6 & -23 & \pm 8 & -75 & \pm 3 \\
\hline Cocaethylene & -1 & \pm 5 & -6 & \pm 5 & -42 & \pm 4 \\
\hline Propranolol & 8 & \pm 6 & 6 & \pm 5 & -27 & \pm 6 \\
\hline AH-7921 & 18 & \pm 15 & 9 & \pm 5 & -26 & \pm 11 \\
\hline Citalopram & 13 & \pm 9 & 14 & \pm 6 & -23 & \pm 7 \\
\hline Fentanyl & 9 & \pm 13 & 10 & \pm 2 & -25 & \pm 0 \\
\hline Midazolam & -3 & \pm 7 & 5 & \pm 10 & -54 & \pm 8 \\
\hline Buprenorphine & 18 & \pm 12 & 23 & \pm 10 & -41 & \pm 14 \\
\hline Carbamazepine & 4 & \pm 6 & 4 & \pm 6 & -11 & \pm 5 \\
\hline EDDP & 45 & \pm 4 & 82 & \pm 5 & 77 & \pm 6 \\
\hline Atomoxetine & 20 & \pm 4 & 22 & \pm 1 & -60 & \pm 7 \\
\hline Nitrazepam & -14 & \pm 12 & -8 & \pm 9 & -79 & \pm 9 \\
\hline Clonazepam & -2 & \pm 11 & -3 & \pm 10 & -65 & \pm 9 \\
\hline Oxazepam & -7 & \pm 8 & -1 & \pm 8 & -36 & \pm 8 \\
\hline Lorazepam & -1 & \pm 10 & -3 & \pm 7 & -46 & \pm 11 \\
\hline Alprazolam & -1 & \pm 6 & 3 & \pm 7 & -23 & \pm 8 \\
\hline Methadone & 3 & \pm 6 & 7 & \pm 8 & -41 & \pm 7 \\
\hline Flunitrazepam & 0 & \pm 5 & 0 & \pm 7 & -64 & \pm 4 \\
\hline Etizolam & -1 & \pm 5 & 2 & \pm 10 & -41 & \pm 7 \\
\hline Phenazepam & 2 & \pm 8 & 3 & \pm 15 & -70 & \pm 9 \\
\hline Diazepam & -5 & \pm 7 & -8 & \pm 10 & -59 & \pm 7 \\
\hline
\end{tabular}


Table S-5

Concentration values (ng/POCIS) for the most commonly detected drugs in the passive samplers

\begin{tabular}{|c|c|c|c|c|c|c|c|c|c|c|}
\hline Analyte & & & & te of collect & $n$ and conc & ntration valt & $s(n g / P O C$ & & & \\
\hline & 29.08 .2013 & 16.09 .2013 & 30.09 .2013 & 15.10.2013 & 29.10.2013 & 15.11.2013 & 02.12 .2013 & 18.18.2013 & 03.01 .2014 & 21.01.2014 \\
\hline Morphine & 34.9 & 39.1 & 48.2 & 22.3 & 22.9 & 18.6 & 25.1 & 22.1 & 16.2 & 30.8 \\
\hline Hydroxycotinine & 18.7 & 14.6 & 17.1 & 44.7 & 28.4 & 40.9 & 24.5 & 36.9 & 27.8 & 31.7 \\
\hline Atenolol & 68.0 & 68.4 & 89.5 & 36.4 & 44.9 & 45.7 & 69.8 & 44.0 & 26.3 & 58.3 \\
\hline Paracetamol & 718.6 & 521.1 & 940.7 & 778.3 & 779.2 & 699.9 & 1086.3 & 1091.3 & 360.5 & 1055.9 \\
\hline MDMA & 33.1 & 33.9 & 48.4 & 25.3 & 25.5 & 4.8 & 49.4 & 32.9 & 20.8 & 26.9 \\
\hline Benzoylecgonine & 78.0 & 77.8 & 85.6 & 43.1 & 52.7 & 46.1 & 86.0 & 58.9 & 39.4 & 58.9 \\
\hline Methoxetamine & 3.3 & 3.5 & 3.8 & 1.5 & 1.9 & 2.0 & 2.6 & 1.4 & 1.2 & 1.8 \\
\hline Metoprolol & 448.5 & 518.4 & 738.6 & 384.7 & 358.3 & 408.5 & 541.5 & 436.6 & 194.7 & 370.0 \\
\hline Cocaine & 106.1 & 110.4 & 133.9 & 69.5 & 68.2 & 72.9 & 121.8 & 85.4 & 38.3 & 68.8 \\
\hline Propranolol & 38.3 & 54.5 & 31.2 & 32.5 & 31.5 & 42.9 & 41.5 & 40.2 & 14.3 & 24.6 \\
\hline Citalopram & 93.8 & 119.1 & 87.6 & 72.7 & 70.2 & 118.0 & 132.8 & 103.7 & 27.2 & 74.0 \\
\hline Carbamazepine & 244.3 & 399.5 & 440.1 & 226.0 & 226.3 & 205.8 & 267.1 & 220.0 & 98.8 & 177.6 \\
\hline EDDP & 97.1 & 169.4 & 139.4 & 103.9 & 81.9 & 91.9 & 114.9 & 78.7 & 20.4 & 74.5 \\
\hline Methadone & 15.1 & 17.2 & 18.9 & 15.8 & 13.1 & 15.2 & 16.9 & 15.1 & 7.0 & 11.3 \\
\hline Oxazepam & 696.9 & 927.5 & 744.8 & 692.4 & 525.6 & 675.3 & 754.7 & 745.0 & 187.1 & 421.1 \\
\hline Diclofenac & 134.2 & 144.8 & 109.9 & 92.2 & 149.7 & 103.5 & 133.9 & 125.3 & 47.4 & 102.8 \\
\hline
\end{tabular}


Table S-6

Concentration (ng/L) values of target compounds in pooled urine samples $(5 \mathrm{~mL})$ from anonymous Norwegian music festivals

\begin{tabular}{cccc}
\cline { 2 - 4 } Compound & Festival 1 & Festival 2 & Festival 3 \\
\hline Atenolol & - & 900 & - \\
Morphine & - & - & 440 \\
Paracetamol* & 3.6 & 52.2 & - \\
Gabapentin & - & - & 20900 \\
Amphetamine & 5840 & 820 & 1780 \\
Methamphetamine & 1360 & - & 60.6 \\
MDMA* & - & - & 4340 \\
MDA & - & 480 & 520 \\
Cocaine & - & 78820 & 14680 \\
Benzoylecgonine & 1340 & & 2360 \\
Cocaethylene & - & - & 720 \\
Citalopram & - & - & - \\
Propranolol & 540 & 7860 & - \\
Carbamazepine & 3520 & 1100 & - \\
Oxazepam & 340 & - & 7080 \\
Methylphenidate & - & - &
\end{tabular}


Table S-7. Occurrence of the identified suspect candidates in the pooled urine samples by UHPLC-QTOF using the suspect database.

\begin{tabular}{|c|c|c|c|c|}
\hline Compound & Number of Identified LE Ions & Retention Time & Elemental Composition & $\mathrm{m} / \mathrm{z}$ \\
\hline 1-Benzylpiperazine (BZP) & 2 & 1.23 & $\mathrm{C} 11 \mathrm{H} 16 \mathrm{~N} 2$ & 176.13134 \\
\hline Hydroxy-metoprolol & 3 & 1.43 & $\mathrm{C} 15 \mathrm{H} 25 \mathrm{NO} 4$ & 283.17834 \\
\hline Caffeine & 3 & 2.08 & $\mathrm{C} 8 \mathrm{H} 10 \mathrm{~N} 4 \mathrm{O} 2$ & 194.08036 \\
\hline Nadolol & 3 & 2.27 & $\mathrm{C} 17 \mathrm{H} 27 \mathrm{NO} 4$ & 309.19399 \\
\hline Metoclopramide & 4 & 3.02 & $\mathrm{C} 14 \mathrm{H} 22 \mathrm{ClN} 3 \mathrm{O} 2$ & 299.14004 \\
\hline Lidocaine & 2 & 3.19 & $\mathrm{C} 14 \mathrm{H} 22 \mathrm{~N} 2 \mathrm{O}$ & 234.1732 \\
\hline O-demethyl-venlafaxine & 3 & 3.2 & $\mathrm{C} 16 \mathrm{H} 25 \mathrm{NO} 2$ & 263.18851 \\
\hline Remifentanil & 3 & 4.37 & $\mathrm{C} 20 \mathrm{H} 28 \mathrm{~N} 2 \mathrm{O} 5$ & 376.1998 \\
\hline Disopramide & 3 & 4.56 & $\mathrm{C} 21 \mathrm{H} 29 \mathrm{~N} 3 \mathrm{O}$ & 339.23104 \\
\hline Zolpidem & 4 & 5.19 & $\mathrm{C} 19 \mathrm{H} 21 \mathrm{~N} 3 \mathrm{O}$ & 307.16845 \\
\hline Venlafaxine & 3 & 5.29 & $\mathrm{C} 17 \mathrm{H} 27 \mathrm{NO} 2$ & 277.20416 \\
\hline Acetildenafil & 5 & 5.37 & $\mathrm{C} 25 \mathrm{H} 34 \mathrm{~N} 6 \mathrm{O} 3$ & 466.26922 \\
\hline Enalapril & 2 & 5.49 & $\mathrm{C} 20 \mathrm{H} 28 \mathrm{~N} 2 \mathrm{O} 5$ & 376.1998 \\
\hline Mepyramine & 2 & 5.52 & $\mathrm{C} 17 \mathrm{H} 23 \mathrm{~N} 3 \mathrm{O}$ & 285.1841 \\
\hline Sildenafil & 2 & 6.19 & $\mathrm{C} 22 \mathrm{H} 30 \mathrm{~N} 6 \mathrm{O} 4 \mathrm{~S}$ & 474.2049 \\
\hline Benzocaine & 2 & 6.5 & $\mathrm{C} 9 \mathrm{H} 11 \mathrm{NO} 2$ & 165.07897 \\
\hline Imipramine & 2 & 7.67 & $\mathrm{C} 19 \mathrm{H} 24 \mathrm{~N} 2$ & 280.19393 \\
\hline Cetirizine & 2 & 8.28 & $\mathrm{C} 21 \mathrm{H} 25 \mathrm{CIN} 2 \mathrm{O} 3$ & 388.15535 \\
\hline Trimipramine & 2 & 8.44 & $\mathrm{C} 20 \mathrm{H} 26 \mathrm{~N} 2$ & 294.20958 \\
\hline Irbesartan & 2 & 9.08 & $\mathrm{C} 25 \mathrm{H} 28 \mathrm{~N} 6 \mathrm{O}$ & 428.23244 \\
\hline Irbesartan & 2 & 9.28 & $\mathrm{C} 25 \mathrm{H} 28 \mathrm{~N} 6 \mathrm{O}$ & 428.23244 \\
\hline Cinnarizine & 2 & 10.48 & $\mathrm{C} 26 \mathrm{H} 28 \mathrm{~N} 2$ & 368.22523 \\
\hline Fluocinonide & 2 & 11.17 & $\mathrm{C} 26 \mathrm{H} 32 \mathrm{~F} 2 \mathrm{O} 7$ & 494.21159 \\
\hline Orlistat & 4 & 13.24 & $\mathrm{C} 29 \mathrm{H} 53 \mathrm{NO} 5$ & 495.39234 \\
\hline
\end{tabular}

(1) Harman, C.; Reid, M.; Thomas, K. V. Environ Sci Technol 2011, 45, 5676-5682. 